doi: $10.15407 /$ ujpe63.01.0049

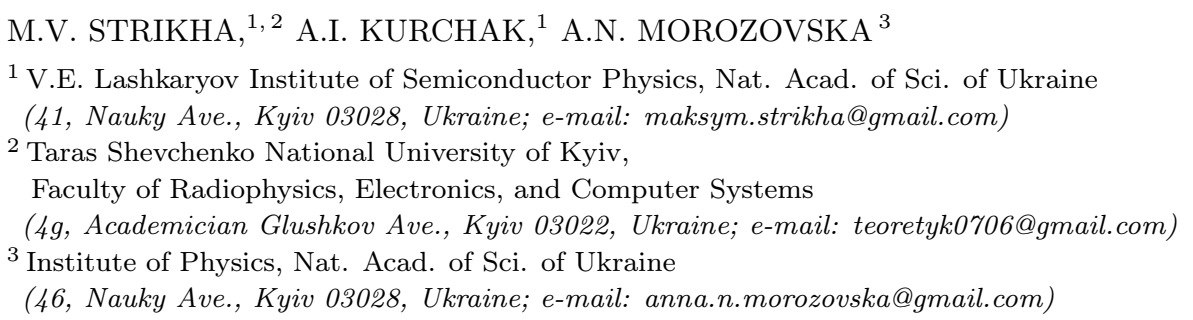

\title{
INFLUENCE OF DOMAIN STRUCTURE IN FERROELECTRIC SUBSTRATE ON GRAPHENE CONDUCTANCE (AUTHORS' REVIEW)
}

\begin{abstract}
This review is devoted to recent theoretical studies of the influence exerted by a domain structure in the ferroelectric substrate on the graphene conductance in the graphene-on-ferroelectric structure. An analytical description of the hysteresis memory effect in the field effect transistor based on this structure, which considers absorbed dipole layers on the free graphene surface and localized states at the graphene-ferroelectric interface, is considered. Some theses of a theory recently developed for the conductance of $p-n$ junctions created by a $180^{\circ}$-ferroelectric domain structure in a single graphene layer (channel) on the ferroelectric substrate, are analyzed, and various current regimes from the ballistic to diffusion one are considered. The size effects in such systems and a possibility to apply the results obtained to improve the parameters of such devices as field effect transistors with a graphene channel, non-volatile ferroelectric memory cells with random access, and sensors, as well as to miniaturize various devices of functional nanoelectronics are discussed.

Ke ywords: graphene-on-ferroelectric structure, domain structure, conductance, field effect
\end{abstract} transistor.

\section{Introduction}

Experimental and theoretical studies of remarkable electromechanical, electrophysical, and transport properties of graphene remained a focus of researchers' attention since the graphene discovery $[1,2]$ till now (see, e.g., works [3-5]). A promising quite feasible way toward the understanding of and control over graphene-based devices (as well as devices, in which other $2 \mathrm{D}$ semiconductors are used) consists in the application of "smart" substrates with additional degrees of functionality: electromechanical, polar, and/or magnetic ones. For instance, graphene on a ferroelectric substrate [6-10], whose spontaneous polarization and domain structure can be controlled by an external electric field [11, 12], can be proposed as such a "smart" system [13].

The structure of this review is as follows. In Section 2, we analyze our recent theoretical works de-

(C) M.V. STRIKHA, A.I. KURCHAK, A.N. MOROZOVSKA, 2018

ISSN 2071-0194. Ukr. J. Phys. 2018. Vol. 63, No. 1 voted to the changes in the graphene channel conductance stimulated by $p-n$ junctions emerging at a single domain wall in the ferroelectric substrate. Various current regimes and rectification effects are also discussed there. In Section 3, we consider the theory of hysteretic phenomena in the graphene-on-ferroelectric structure. In Section 4, the dynamics of $p$ $n$ junctions in a graphene channel induced by the motion of ferroelectric domain walls is analyzed. Section 5 contains a discussion concerning the possibilities of graphene separation and stretching induced by the piezoelectric effect in ferroelectric domains. A brief summary of the presented results is made in Conclusions.

\section{Conductance of a Graphene Channel with a $\boldsymbol{p}-\boldsymbol{n}$ Junction at a Ferroelectric Domain Wall}

The presence of a domain structure in a ferroelectric substrate can lead to the formation of $p-n$ junctions in graphene $[11,12]$, which are located above 


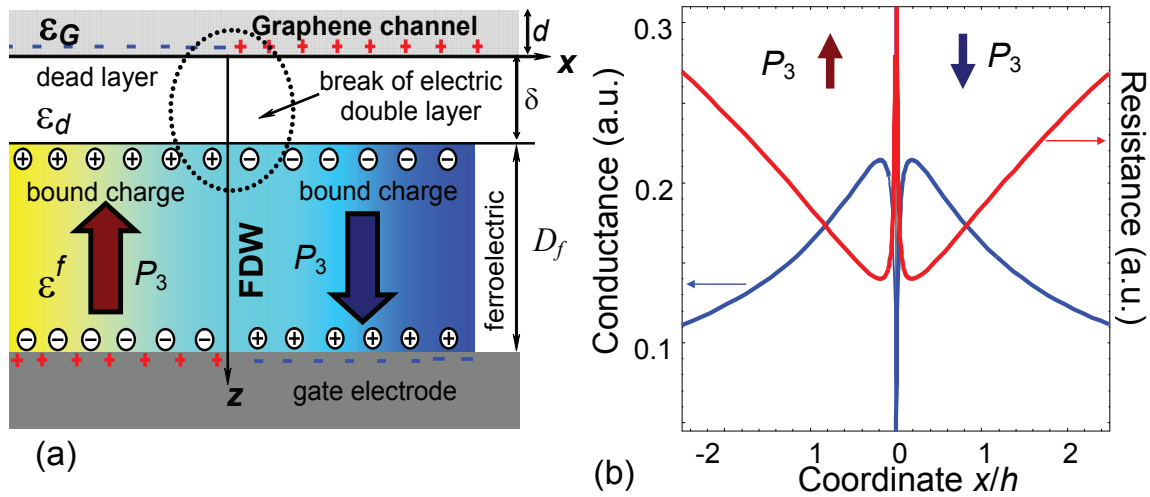

Fig. 1. $180^{\circ}$-FDW structure near the ferroelectric surface in the graphene-on-ferrroelectric structure. The discontinuity of the electric double layer consisting of screening and bound charges creates a depolarization electric field that penetrates into the gap (a). Graphene conductance (left vertical axis) and resistance (right vertical axis) $x$-distributions caused by the FDW located at $x=0$ in the graphene-on-ferrroelectric heterostructure (b). Reproduced from work [A.N. Morozovska, E.A. Eliseev, M.V. Strikha. Appl. Phys. Lett. 108, 232902 (2016)] with permission from AIP Publishing

the domain walls in the ferroelectric substrate [1418]. Note that the unique properties of the $p-n$ junction in graphene have been realized much earlier by the multiple-gate doping of a graphene channel by electrons and/or holes [19-21]. Later, they have been studied both theoretically $[22,23]$ and experimentally [24-26]. Hinnefeld et al [11] and Baeumer et al [12], using ferroelectric substrates, created a $p-n$ junction in graphene by imposing a graphene sheet on a $180^{\circ}$-ferroelectric domain wall (FDW). Due to the charge separation by an electric field at the FDW-surface junction, there arise $p-n$ junctions in graphene $[27,28]$.

Note that an elastic strain can change the band structure in graphene (e.g., via the deformation potential) and open a band gap [4,5,29-31]. We showed [18] that a piezoelectric displacement of the ferroelectric domain surfaces can lead to a stretching and separation of graphene sections at the steps between elongated and shortened domains. The graphene separation at FDWs induced by the piezo-effect can change the graphene channel conductance. Below, we briefly review the results of works [14-18] and discuss their correlation with available experimental and other theoretical works.

The semiquantum and semiphenomenological analytical models were developed for various types of carrier transport (ballistic, diffusive, etc.) in a singlelayer graphene channel at the $180^{\circ}$-FDW $[14,15]$. Specifically, the influence of a ferroelectric domain wall on the ballistic conductance of the graphene channel was studied in our work [14] in the WentzelKramers-Brillouin (WKB) approximation. In particular, we considered a graphene channel separated from a ferroelectric layer with the FDW structure by an ultra-thin dielectric layer (a physical gap or a ferroelectric dead layer). The ferroelectric layer makes a perfect electric contact with the gate electrode (see Fig. 1, a).

A pronounced broadening of the FDW appears near the surface $[14,32]$ in order to decrease a depolarizing electric field generated by uncompensated bound polarization charges localized in the thin subsurface layer of ferroelectric. Despite the broadening, the stray electric field is still strong enough to induce a $p-n$ junction in the graphene channel. A potential barrier for electrons and holes emerges in the $p-n$ junction, when a positive (negative) voltage is applied to the left (right) contact of the channel. The probability $w$ for an electron, which is characterized by the wave vector $\mathbf{k}$ directed at the angle $\vartheta$ with respect to the $x$-axis in the $n$-region, to pass into the $p$-region can be calculated using the scheme presented in work [20]. With an allowance for the linear graphene band spectrum

$E\left(k_{x}, k_{y}\right)=v_{\mathrm{F}} \hbar \sqrt{k_{x}^{2}+k_{y}^{2}}$,

the electron kinetic energy at the junction center, $x=0$, equals $v_{\mathrm{F}} \hbar \sqrt{k_{x}^{2}+k_{y}^{2}}$, where the $y$-component $k_{y}=k_{\mathrm{F}} \sin \vartheta$ of the momentum is conserved, and $v_{\mathrm{F}} \approx 10^{6} \mathrm{~m} / \mathrm{s}$ is the so-called Fermi velocity of 
the electron (it is determined by the energy of $\sigma$ bonds between carbon atoms in the graphene plane $[3])$. Therefore, the $x$-component is determined by the expression

$k_{x}(x)=\sqrt{\left(e \varphi(x, 0) / v_{\mathrm{F}} \hbar\right)^{2}-\left(k_{\mathrm{F}} \sin \vartheta\right)^{2}}$.

The classically allowed region of electron motion is determined by the inequality $e \varphi(x, 0)>v_{\mathrm{F}} \hbar k_{y}$, where $\varphi(x)$ is the spacially dependent potential of the $p-n$ junction barrier for an electron, which means that the electron cannot overpass the turning point at the distance $l_{x}$ from the center of the junction located at the FDW.

For small angles, $|\vartheta| \ll 1$, the probability $P^{*}$ can be estimated quasiclassically in the WKB approximation as $P^{*} \approx e^{-2 S / \hbar}$, where $S=i \hbar \int_{-l_{x}}^{l_{x}} k_{x}(x) d x$. The conductance value per unit of the $p-n$ junction width was evaluated by the formula [14]

$G_{p n}^{\text {ball }} \approx \frac{e^{2} k_{\mathrm{F}}}{2 \pi^{2} \hbar} \sqrt{\frac{\pi}{P_{x}}}$,

where

$k_{\mathrm{F}}=\frac{\pi^{2} \epsilon_{0} \epsilon_{33}^{\mathrm{f}}}{e^{2}} \frac{\hbar v_{\mathrm{F}} Q}{\delta^{*} \ln 3}$

is the Fermi wave vector,

$Q=\frac{e\left(P_{S} / \epsilon_{0}\right)}{\epsilon_{33}^{\mathrm{f}}+\gamma \epsilon_{\mathrm{d}}} \frac{2 \gamma \delta^{*} \ln 3}{\pi \hbar k_{\mathrm{F}} v_{\mathrm{F}}}$

is a dimensionless factor,

$P_{x} \approx \pi^{3} \epsilon_{0} \epsilon_{33}^{\mathrm{f}} \hbar v_{\mathrm{F}} / e^{2}$

is the spontaneous polarization, $e$ the elementary charge, $\epsilon_{0}$ the universal dielectric constant, $\epsilon_{\mathrm{d}}$ the relative permittivity of a dielectric layer (it is equal either to the background permittivity of ferroelectric in the case of dead layer or to unity in the case of physical gap), $\gamma=\sqrt{\epsilon_{33}^{\mathrm{f}} / \epsilon_{11}^{\mathrm{f}}}$ is the anisotropy factor of ferroelectric, $\epsilon_{i i}^{\mathrm{f}}$ are the components of the relative permittivity of the ferroelectric substrate $\left(\epsilon_{33}^{\mathrm{f}}\right.$ is the permittivity of ferroelectric along the $z$-direction, i.e. normal to the graphene plane; and $\epsilon_{11}^{\mathrm{f}}$ is the permittivity of ferroelectric along the $x$-direction, i.e. along the channel), $\delta^{*}$ is the sum of the dielectric layer thickness $\delta$ and the effective screening length in graphene $[14,33]$.

Since the wave vector $k_{\mathrm{F}} \equiv \sqrt{\pi n_{2 \mathrm{D}}}$, Eq. (2.1) can be rewritten in the form [14]

$G_{p n}^{\mathrm{ball}} \cong \frac{e^{2}}{\pi \hbar} \sqrt{\frac{\alpha}{\epsilon_{33}^{\mathrm{f}}} \frac{c}{\pi v_{\mathrm{F}}} n_{2 \mathrm{D}}}$,

ISSN 2071-0194. Ukr. J. Phys. 2018. Vol. 63, No. 1 where $\alpha=e^{2} / 4 \pi \epsilon_{0}(\hbar c)$ is the fine-structure constant, and $c$ is the light velocity in vacuum. To within the dimensionless factor $\alpha=e^{2} / 4 \pi \epsilon_{0}(\hbar c) \sim 0.1$, Eq. (2.2) coincides with the textbook expression for the ballistic conductance of a graphene channel per unit width $[3,34]$

$G=2 e^{2} \sqrt{n_{2 \mathrm{D}}} / \hbar$.

However, Eq. (2.2) differs from Eq. (2) in work [20], because the concentration of $2 \mathrm{D}$-carriers in graphene in the graphene-on-ferroelectric structure, $n_{2 \mathrm{D}}$, is governed by the spontaneous polarization of ferroelectric,

$n_{2 \mathrm{D}}(x) \approx \frac{2 \gamma \epsilon_{33}^{\mathrm{f}}\left(P_{S} / e\right)}{\epsilon_{33}^{\mathrm{f}}+\gamma \epsilon_{\mathrm{d}}}$.

The nominal resistance $R_{n p}$ is reciprocal to the conductance [Eq. (2.2), i.e. $R_{n p}=1 /\left(W G_{p n}^{\text {ball }}\right)$, where $W$ is the $p-n$ junction width.

The conductance and resistance profiles arising near the FDW are shown in Fig. 1, b. As one can see, the width of the $p-n$ junction region is about $2 h$. Since $\sigma_{0}=e^{2} /(2 \pi \hbar)$ is a conductance quantum, the ratio $W G_{p n}^{\text {ball }} / \sigma_{0}$ gives us a notion about the number of conductivity modes. This result coincides by order with that calculated in work [35].

The estimation of the concentration caused by ferroelectric dipoles leads to values of about $10^{19} \mathrm{~m}^{-2}$, which is two orders of magnitude higher than the highest possible ones for gated graphene on the $\mathrm{SiO}_{2}$ substrate (this limit is determined by the dielectric breakdown field). Therefore, the graphene $p-n$ junction at the FDW should have been characterized by rather a high ballistic conductance and a low resistance. However, the factor $1 /\left(\epsilon_{33}^{\mathrm{f}}\right)^{1 / 2}$, where $\epsilon_{33}^{\mathrm{f}}$ can be rather large for ferroelectric substrates, enters Eq. (2.2) for the conduction of a $p-n$ junction only if a direct voltage is applied between the source and drain electrodes (the electron moves through the $p-n$-junction barrier). Otherwise, this factor is absent (the electron does not feel the barrier when moving in the opposite direction). As a result, a graphene $p$ $n$ junction at the ferroelectric domain could be an excellent rectifier with a conductance ratio of about 10 between the direct and reverse polarities of the applied voltage.

However, the performance of the majority of realistic graphene devices depends on the electron mean 


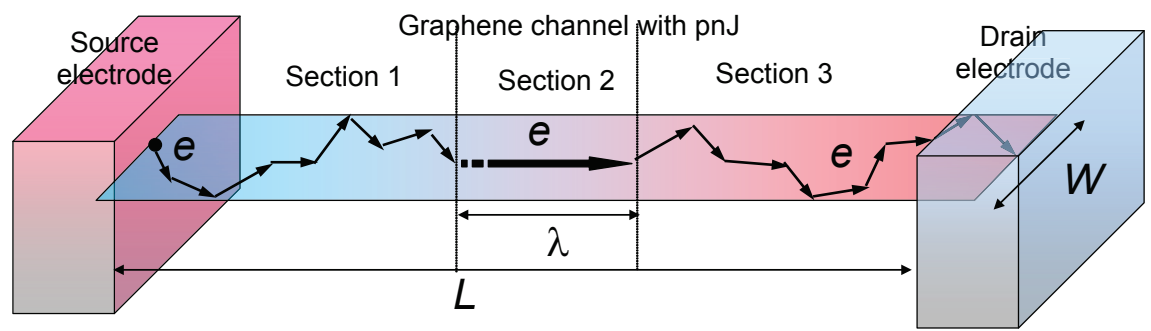

Fig. 2. Conventional partition of a graphene channel with a $p-n$ junction (pnJ) into three sections. The current is diffusive in sections 1 and 3 , and ballistic in section 2 . Reproduced from work [M.V. Strikha, A.N. Morozovska. J. Appl. Phys. 120, 214101 (2016)] with permission from AIP Publishing

free path $\lambda \sim 50 \div 250 \mathrm{~nm}$, so that they operate in the diffusion regime [15]. Since the electron mean free path $\lambda$ in the graphene channel is usually much longer than the a typical thickness $w$ of the uncharged domain wall in a proper ferroelectric (about 1-10 nm) [36], one can divide a graphene channel of the length $L$ between the source and the drain into three sections: a section with the length $\lambda$ containing the $p-n$ junction and two sections located on the both sides from the junction with the total length $L-\lambda$ (see Fig. 2).

The resulting conductance of the sample is governed by the evident expression [15]

$G^{\text {total }}=\frac{W}{\frac{1}{G^{\text {diff }}}+\frac{1}{G^{\text {ball }}}}$.

The conductance is proportional to the graphene channel width $W$, and the textbook relation

$G^{\text {diff }}=\frac{\lambda}{L+\lambda} G^{\text {ball }}$

is valid (see, e.g., works $[38,48]$ ). The change of the voltage polarity between the contacts does not change the conductance of sections 1 and 3. On the contrary, the conductance per unit width in section 2 is described by the expression

$G^{\text {ball }}=\frac{2 e^{2}}{\hbar \pi^{3 / 2}} \sqrt{n_{2 \mathrm{D}}}$

for the positive voltage polarity and the expression

$G_{p n}^{\mathrm{ball}} \cong \frac{e^{2}}{\pi \hbar} \sqrt{\frac{\alpha}{\epsilon_{33}^{\mathrm{f}}} \frac{c}{\pi v_{\mathrm{F}}} n_{2 \mathrm{D}}}$

for the negative one [see Eq. (2.2) and work [15]]. The permittivity $\epsilon_{33}^{\mathrm{f}}$ of ferroelectric in the direction normal to the graphene plane can be rather high: about
500 for piezoceramics (PZT) or even higher (about 5000) for relaxor ferroelectrics. Thus, the conductance ratio for different voltage polarities equals

$\frac{G_{+}^{\text {total }}}{G_{-}^{\text {total }}}=\frac{\beta(L+\lambda)}{\beta(L+\lambda)+\lambda}$.

The parameter $\beta$ depends on the dielectric permittivity,

$\beta\left(\epsilon_{33}^{\mathrm{f}}\right)=\sqrt{\pi \alpha c /\left(4 \epsilon_{33}^{\mathrm{f}} v_{\mathrm{F}}\right)}$,

and the electron mean free path $\lambda$ depends on the concentration $n_{2 \mathrm{D}}$ or - equivalently, since $n_{2 \mathrm{D}} \sim$ $\sim \pi\left(P_{S} / e\right)[15,33]$ - on the ferroelectric polarization. If the scattering of electrons in the graphene channel by charged impurities in ferroelectric dominates (this is the most common case for real graphene-based operational devices; see, e.g., work $[3]), \lambda\left(n_{2 \mathrm{D}}\right) \sim \sqrt{n_{2 \mathrm{D}}}$. In the case of short ballistic channel, $L \ll \lambda$, we have $G^{\text {total }} \approx G^{\text {ball }}$. In the opposite limit (a long diffusion channel, $L \gg \lambda$ ), we obtain $G_{+}^{\text {total }} \approx G_{-}^{\text {total }}$, so that the rectifying properties of the $p-n$ junction disappear.

The dependences of the ratio $G_{+}^{\text {total }} / G_{-}^{\text {total }}$ on the ratio $L / \lambda$ calculated for various ferroelectric substrates (relaxor, $\mathrm{Pb}(\mathrm{Zr}, \mathrm{Ti}) \mathrm{O}_{3}, \mathrm{BaTiO}_{3}, \mathrm{LiTaO}_{3}$, and $\left.\mathrm{LiNbO}_{3}\right)$ are exhibited in Fig. 3. As one can see, small $G_{+}^{\text {total }} / G_{-}^{\text {total }}$-values can be obtained at $L / \lambda \lesssim 1$ for ferroelectric substrates with a high permittivity. The expected result can be implemented for channels with a submicronic length in the case of the comparable values of the electron mean free path. The situation corresponds to the transient current regime from the diffusion to ballistic one. Note that the distance between the parallel FDWs is generally much 
larger than the electron mean free path, which enables a micron-length channel with contacts located near only one FDW to be fabricated. For ferroelectrics with an extremely high permittivity, such as relaxors or $\mathrm{PbZr}_{x} \mathrm{Ti}_{1-x} \mathrm{O}_{3}$ with the $\mathrm{Zr}$ content $x$ near the morphotropic phase boundary $x=0.52$, the ratio $G_{+}^{\text {total }} / G_{-}^{\text {total }}$ can be essentially smaller than unity in the case of pronounced current diffusion regime as well. This fact allows us to consider ferroelectric substrates with an ultra-high permittivity as excellent candidates for the fabrication of novel rectifiers based on the graphene $p-n$ junction.

\section{Hysteretic Phenomena in the Graphene-on-Ferroelectric Structure}

\subsection{Theoretical formalism}

The dependence of the graphene channel conductance on the gate voltage $V_{g}$ is generally considered to be excellently symmetric, $G\left(V_{g}\right)=G\left(-V_{g}\right)$ [3]. However, this relation is valid for specially treated high-quality graphene surfaces only. In many real imperfect structures with absorbed dipoles at free graphene centers, localized states at the graphene-substrate interface, and so forth, this symmetry disappears. Moreover, the dependence $G\left(V_{g}\right)$ can get a hysteretic form (see, e.g., original works $[36,59,60]$ and reviews $[61,62])$. This hysteresis can have loops with opposite directions, and needs special consideration with allowance for various competitive physical factors.

In the framework of the theoretical formalism used in work [16], let us consider a conducting graphene channel, which is placed on a dielectric or ferroelectric substrate (see Fig. 4). The concentration of $2 \mathrm{D}$ carriers in the channel is governed by several factors. These are the time-dependent gate voltage $V_{g}(t)$, the polarization of dipoles in the ferroelectric substrate, the polarization of dipoles (e.g., absorbed water molecules) on the graphene surface, and the trapped charge carriers localized at the graphenesubstrate interface. Taking all the abovementioned factors into account, the graphene channel resistivity can be presented in the form [16]

$\rho\left[V_{g}, P_{s} P_{f}, T\right] \approx \frac{1}{\sigma\left(V_{g}, P_{s} P_{f}, T\right)}+\frac{1}{\sigma_{\mathrm{intr}}(T)}+\frac{1}{\sigma_{\min }}$,

where $T$ is the temperature, $P_{s}(t)$ is the polarization of dipoles absorbed on the graphene surface, and

ISSN 2071-0194. Ukr. J. Phys. 2018. Vol. 63, No. 1

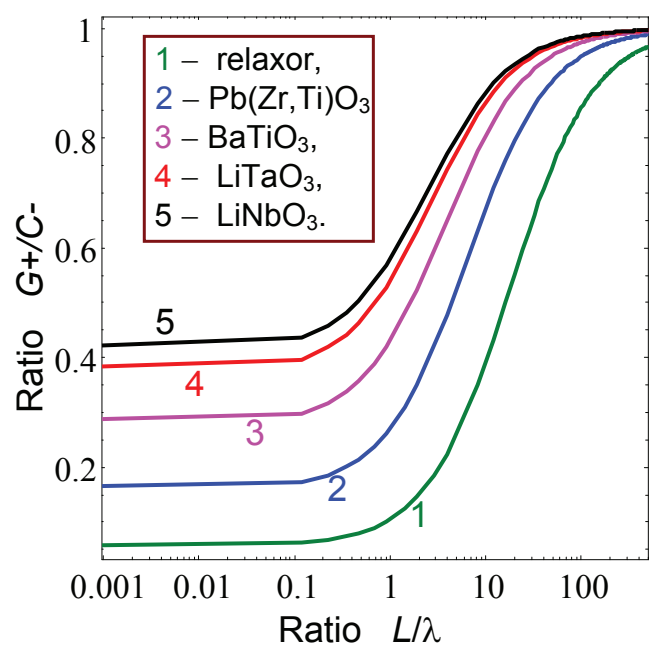

Fig. 3. Ratio $G_{+}^{\text {total }} / G_{-}^{\text {total }}$ between the total pnJ conductances at opposite gate voltage polarities as a function of the ratio $L / \lambda$ between the graphene channel length and the charge carrier mean free path. Calculations were carried out for room temperature and various ferroelectric substrates described by different values of the parameter $\beta\left(\epsilon_{33}^{\mathrm{f}}\right)$ [see Eq. (2.5)]. The dielectric permittivity $\epsilon_{33}^{\mathrm{f}}=5000$ or higher for ferroelectric relaxor (1), 500 for $\mathrm{Pb}(\mathrm{Zr}, \mathrm{Ti}) \mathrm{O}_{3}$ (2), 120 for $\mathrm{BaTiO}_{3}$ (3), 50 for $\mathrm{LiTaO}_{3}$ (4), and 36 for $\mathrm{LiNbO}_{3}$ (5). Reproduced from work [M.V. Strikha, A.N. Morozovska. J. Appl. Phys. 120, 214101 (2016)] with permission from AIP Publishing

$P_{f}(t, T)$ is the temperature-dependent ferroelectric polarization. In the usual case of graphene under ambient conditions, the scattering of ionized impurities by the substrate dominates [23], so that the conductivity of the $2 \mathrm{D}$ graphene channel equals

$\sigma\left(V_{g}, P_{s} P_{f}, T\right)=\operatorname{e\mu n}\left(V_{g}, P_{s} P_{f}, T\right)$,

where $n\left(V_{g}, P_{s}, P_{f}\right)$ is the surface concentration of $2 \mathrm{D}$ carriers induced by the gate mixed doping, the dipoles absorbed on the surface, and the dipoles in the ferroelectric; and $\mu$ is the carrier mobility. The second term on the right-hand side of Eq. (3.1) is the intrinsic graphene conductivity, $\sigma_{\mathrm{intr}}(T)=e \mu n_{\mathrm{intr}}(T)$, where

$n_{\text {intr }}(T)=\frac{2\left(k_{B} T\right)^{2}}{\pi\left(\hbar v_{\mathrm{F}}\right)^{2}}$.

The third term on the right-hand side of Eq. (3.1) corresponds to the minimum quantum conductivity, $\sigma_{\min } \approx \frac{4 e^{2}}{\hbar}$, which nevertheless becomes significant at low $T$.

There are localized states at the graphene-substrate interface. In the voltage interval $V_{g}(t)<V_{T 1}$, 


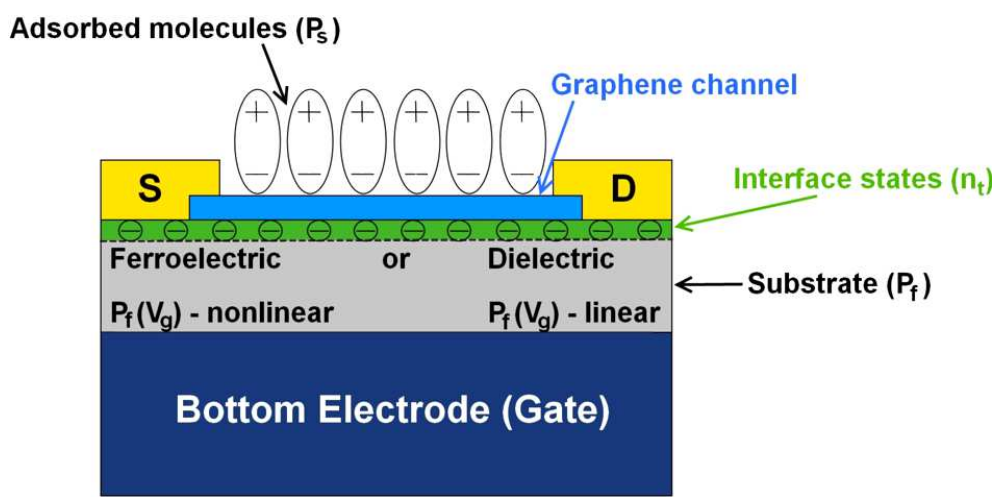

Fig. 4. Graphene sheet (channel) placed on a dielectric or ferroelectric substrate. Dipoles (e.g., polar water molecules) can be adsorbed on the free graphene surface. Charge carriers from the channel can be trapped by centers localized at the graphenesubstrate interface. Reproduced from work [A.I. Kurchak, A.N. Morozovska, M.V. Strikha. J. Appl. Phys. 122, 044504 (2017)] with permission from AIP Publishing

where $V_{T 1}$ is the voltage, at which the electrons from the graphene channel begin to occupy the interface states, $E_{\mathrm{F}}\left(V_{T 1}\right)=E_{T 1}$, the $2 \mathrm{D}$ concentration of electrons in the graphene channel is given by the capacitor formula $[37,38]$

$n\left(V_{g}, t\right)=\frac{\kappa V_{g}(t)}{4 \pi e d}$

where $\kappa$ is the dielectric permittivity of the substrate, and $d$ is the substrate thickness. The gate voltage

$V_{T 1}=\frac{4 \pi e d}{\kappa} \frac{E_{T 1}^{2}}{\pi \hbar^{2} v_{\mathrm{F}}^{2}}$

corresponds to the start of interface state occupation by electrons from the graphene channel, whereas the voltage

$V_{T 2}=\frac{4 \pi e d}{\kappa} \frac{E_{T 2}^{2}}{\pi \hbar^{2} v_{\mathrm{F}}^{2}}+\frac{4 \pi e d n_{T}}{\kappa}$

to the termination of this process, i.e. all localized interface states are occupied [38]. In the voltage interval $V_{T 1} \leq V_{g}<V_{T 2}$,

$n=\frac{E_{T 1}^{2}}{\pi \hbar^{2} v_{\mathrm{F}}^{2}}$.

Finally, in the voltage interval $V_{g} \geq V_{T 2}$, when all interface states are occupied by electrons, the concentration of free electrons in the channel is described by the evident formula

$n\left(V_{g}\right)=\frac{\kappa V_{g}(t)}{4 \pi e d}-n_{T}$.
Let us consider the polarization of dipoles in the ferroelectric substrate and at the graphene surface. If $E_{\mathrm{F}} \leq E_{T 1}$, similarly to works $[37,38]$, we obtain

$n\left(V_{g}, P_{s}, P_{f}\right)=\frac{\kappa V_{g}(t)}{4 \pi e d}+\frac{P_{s}(t)+P_{f}(t, T)}{e}$.

The temperature-dependent spontaneous ferroelectric polarization can be described by the expression [36]

$P_{f}(t, T)=P_{f}(T) \tanh \left(s_{f}\left(V_{g}(t)-V_{c}\right)\right)$,

where $V_{c}=d$ is the coercive voltage equal to the product of the coercive field $E_{c}$ and the substrate thickness $d$, and $s_{f}$ is the "sharpness" of the ferroelectric switching. The spontaneous polarization of the dipoles absorbed on the graphene surface equals

$P_{s}(t)=P_{s} \frac{1-\tanh \left(s_{s}\left(V_{g}(t)-V_{s}\right)\right)}{2}$,

where $V_{s}$ is a critical voltage that ultimately destroys the polarization, $s_{s}$ is a parameter reflecting the "sharpness" of the dipole switching. At the backward sweep, the polarizations $P_{f}$ and $P_{s}$ are

$$
\begin{aligned}
& P_{f}(t, T)=P_{f}(T) \tanh \left(s_{f}\left(V_{g}(t)+V_{c}\right)\right), \\
& P_{s}(t)=P_{s}\left[1-\exp \left(-\frac{\left.t\left(V_{s}\right)-t\right)}{\tau}\right)\right]
\end{aligned}
$$

where $t\left(V_{s}\right)$ is the time moment corresponding to the complete suppression of $P_{s}$ by the critical gate voltage $V_{c}$, and $\tau$ the dipole relaxation time, which can equal

ISSN 2071-0194. Ukr. J. Phys. 2018. Vol. 63, No. 1 

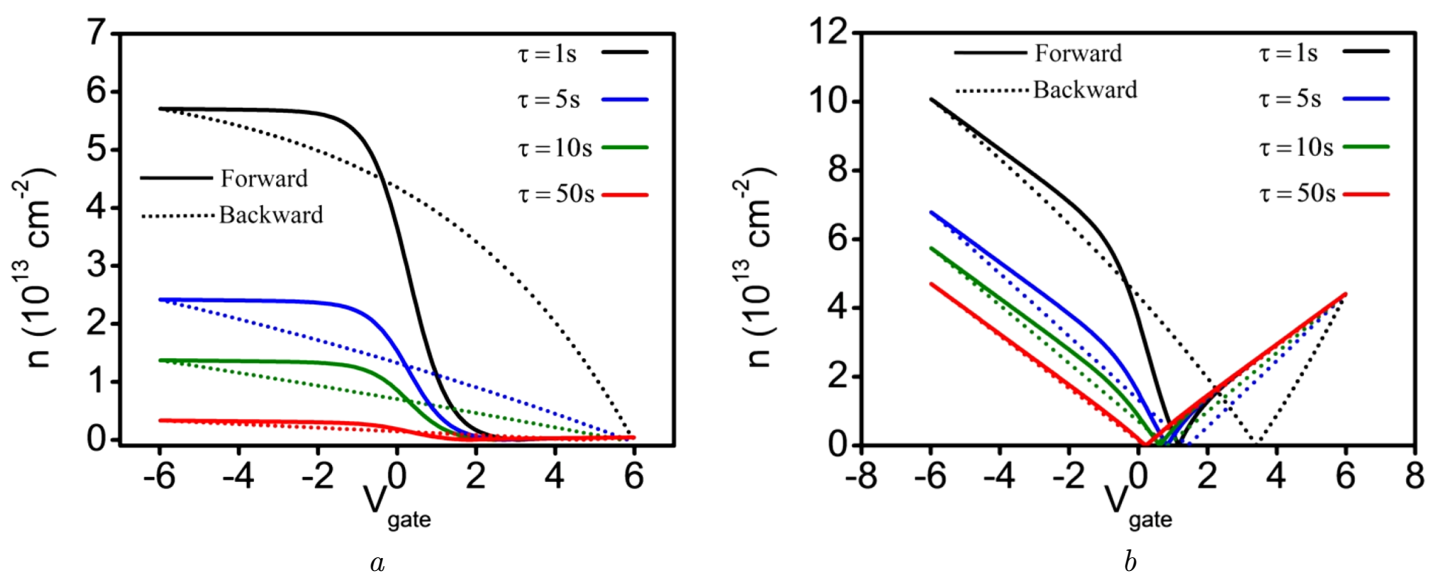

Fig. 5. Dependences of the charge carrier concentration in the graphene channel located on the $\mathrm{SiO}_{2}(a)$ and $\mathrm{PZT}(b)$ substrates on the gate voltage for various surface dipole relaxation times $\tau$. The forward and backward sweeping dependences are presented by solid and dotted curves, respectively. The time dependence of the gate voltage $V_{g}(t)$ is saw-like. Reproduced from work [A.I. Kurchak, A.N. Morozovska, M.V. Strikha. J. Appl. Phys. 122, 044504 (2017)] with permission from AIP Publishing

about several seconds [39]. Equations (3.3)-(3.5) are written for the case where the absorbed dipoles recover immediately after the switching to the backward sweep. The equations above are valid in the case where the lifetime of the carriers trapped by the interface states is much longer than the switching time. The validity of this approximation for graphene on the PZT substrate was demonstrated experimentally in work [40].

The nonlinear response of a perfect ferroelectric substrate can be described using the Ginzburg-Landau-Khalatnikov relaxation equation [33, 41, 42]

$\Gamma \frac{d P(t)}{d t}=\alpha(T) P(t)+\beta P^{3}(t)+\gamma P^{5}(t)-E(\omega, t)$,

where $\alpha, \beta$, and $\gamma$ are the coefficients in the Landau potential expansion.

\subsection{Gate control of the carrier concentration in the graphene channel}

\subsubsection{Influence of surface}

dipoles on graphene charge carriers

Dipoles can be adsorbed on the graphene surface (e.g., adsorption of water molecules was studied experimentally in works $[43-45])$. In this case, $P_{s} \neq 0$, $P_{f}=0$, and $n_{T}=0$. The dipoles shift the electroneutrality point into the positive $V_{g}$-interval [16], and the conductivity of the graphene channel at the zero gate voltage is of the hole type. In this case, the charge carrier concentration in the graphene channel is determined by both the gate doping and the polarization of absorbed dipoles. The latter, in turn, depends on the external electric field created by the gate voltage. The estimates [16] showed that the conductivity of the graphene channel can be governed by the time-dependent polarization of the dipoles.

Figure 5, $a$ illustrates the dependence of the charge carrier concentration on the gate voltage in the graphene channel on a $\mathrm{SiO}_{2}$ substrate at various surface dipole relaxation times $\tau$. As was marked above, the conductivity of the graphene channel at the initial moment (at $V_{g}=0$ ) is determined by holes. However, the electric field generated by the gate potential destroys the polarization of dipoles at some critical $V_{g^{-}}$ value. Solid lines correspond to the second forward sweep, i.e. the polarization disappears after the first cycle of its recovery. The polarization start to renew at the backward sweep. The recovered value of polarization within a switching period is different for different relaxation times $\tau$, but it recovers almost completely during a switching period, if the relaxation time is short. The direction of the corresponding hysteresis loop can be opposite to the so-called direct direction created by ferroelectric dipoles ("antihysteresis").

\subsubsection{Influence of ferroelectric substrate}

on graphene charge carriers at low gate voltages

$\mathrm{Pb}\left(\mathrm{Zr}_{x} \mathrm{Ti}_{1-x}\right) \mathrm{O}_{3}$ (PZT) is a material of choice for GFETs $[40,46,47]$, because its relative dielectric per- 

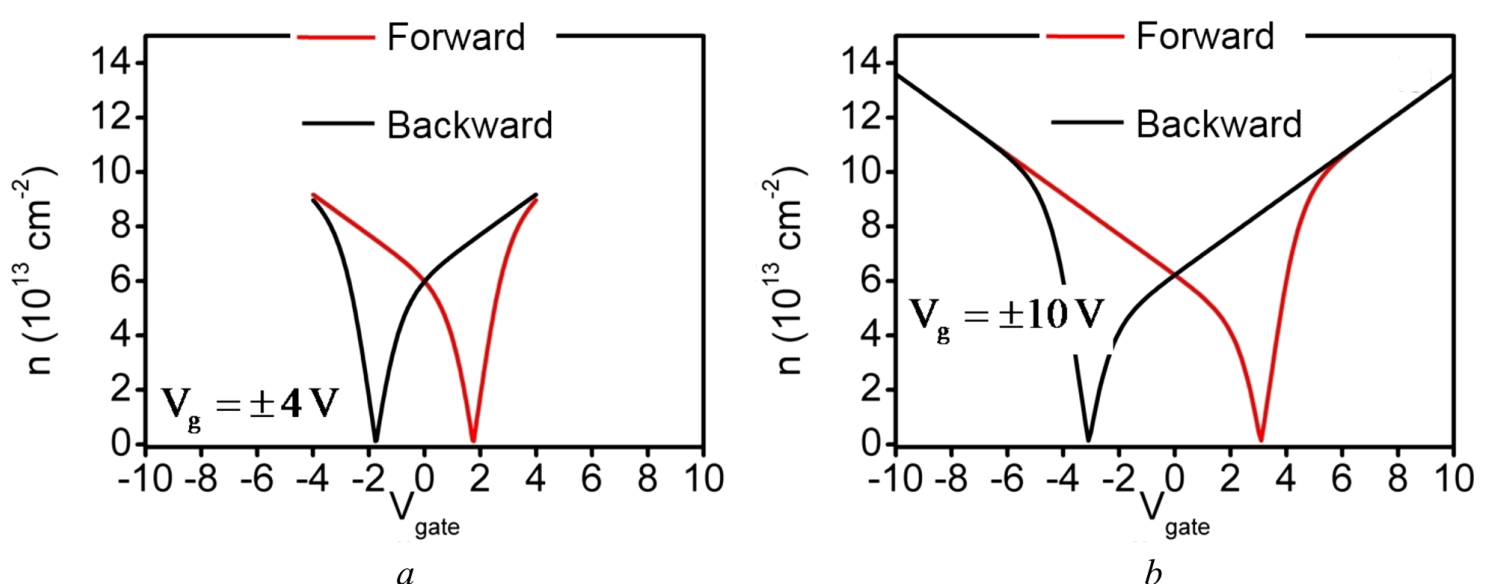

Fig. 6. Dependences of the charge carrier concentration in the graphene channel located on a perfect ferroelectric substrate on the gate voltage for various gate voltage amplitudes $V_{g}^{\max }= \pm 4(a)$ and $\pm 10 \mathrm{~V}(b)$. Reproduced from work [A.I. Kurchak, A.N. Morozovska, M.V. Strikha. J. Appl. Phys. 122, 044504 (2017)] with permission from AIP Publishing

mittivity varies and can be very high near the morphotropic phase boundary (at $x=0.52$ ). Let us analyze the calculation results obtained for the conductance of the graphene channel on the PZT substrate. Taking into account that the PZT response is not hysteretic for the electric fields lower than the coercive field $E_{c}[37,38]$, we will consider a relatively narrow gate voltage interval from $-8 V$ to $+8 \mathrm{~V}$, in which the linear response approximation for $P\left(V_{g}\right)$ is valid. Therefore, the further analysis is similar to that in Section 33.2, but the ferroelectric substrate permittivity is much higher than unity.

Figure 5, $b$ shows the charge carrier concentration in graphene calculated for various dipole relaxation times $\tau$. The graphene channel on the PZT substrate possesses the hole conductivity at $V_{g}=0$, similarly to the case of the $\mathrm{SiO}_{2}$ substrate. For positive gate voltages, the hole concentration decreases and finally reaches the electro-neutrality point, where the graphene valence band becomes completely occupied by electrons, whereas the conduction band is empty. The graphene channel conductivity switches to the electron type as the gate voltage increases further.

\subsubsection{Influence of ferroelectric substrate} on graphene charge carriers at high gate voltages

Figure 6 exhibits the carrier concentration in the graphene channel on a perfect ferroelectric substrate as a function of the gate voltage, $n\left(V_{g}\right)$, at room tem- perature. Figure 3 demonstrates a pronounced hysteresis in the $n\left(V_{g}\right)$ dependence corresponding to the ferroelectric polarization reversal induced by the polarity change of the gate voltage from the maximum negative value $-V_{g}^{\max }$ to the maximum positive one $+V_{g}^{\max }$. In contrast to the case shown in Fig. 2, the hysteresis in Fig. 3 is caused by two different stable states of the ferroelectric polarization. Therefore, it can be used to fabricate nonvolatile memory cells $[46,47]$. However, the corresponding switching times are comparable with the times of reversal of ferroelectric domains, which imposes strict limitations on the operating frequency for thick ferroelectric slabs $\left(10^{4}-10^{6} \mathrm{~Hz}\right)$. But those times can be several orders of magnitude shorter (about $10^{-9} \mathrm{~s}$ ) for thin ferroelectric films with a thickness less than $100 \mathrm{~nm}$ [42].

\subsubsection{Comparison with real experiments}

In order to compare the obtained results with those of real experiments, let us consider two competitive mechanisms that govern the concentration of charge carriers in graphene. The ferroelectric dipoles are responsible for one of them, and the absorbed surface dipoles for the other one. Figure 7 exhibits experimental [48] and theoretical results concerning the electro-neutrality point position as a function of the gate voltage sweeping rate for the GFET with a grapene sheet on the $140-\mathrm{nm} \mathrm{PbZr}_{0.2} \mathrm{Ti}_{0.8} \mathrm{O}_{3} / 60-\mathrm{nm}$ $\mathrm{SrRuO}_{3} / \mathrm{SrTiO}_{3}(001)$ heterostructure. From Fig. 7, one can see that the calculated values are in a reason- 
able agreement with the experimental data [48]. The discrepancy between the theoretical and experimental dependences can be induced by the presence of interface states between graphene and ferroelectric, as well as by a chemical doping of graphene during its fabrication.

We analyzed the origin of the hysteretic graphene conductance dependence on the gate voltage for graphene imposed on various substrates. It was demonstrated that an increase of the gate voltage sweeping rate $d V_{g} / d t$ leads to the disappearance of the hysteresis for the GFET on dielectric or ferroelectric substrates, if the gate voltage is less than the coercive one, $V_{g}<V_{c}$. An increase of the gate voltage rate causes the transition from the anti-hysteresis to the ferroelectric hysteresis. These results are in qualitative and quantitative agreement with the experimental data [48].

Note that the results of work [16] were obtained using a number of approximations and simplifications. However, the actual physical origin of the dipole bonding to the graphene surface was not taken into account, as well as a possible relaxation time spectrum. The fact that the trapping of charge carriers by the surface states depends on the gate voltage switching frequency was also not taken into consideration. A description of the mentioned effects is possible provided a complete understanding of the physical mechanism of trapping and requires further studies.

\section{Dynamics of $p-n$ Junctions} in a Graphene Channel Induced by the Motion of Ferroelectric Domain Walls

\subsection{Theoretical formalism}

In Section 2, we considered the case of fixed FDWs. However, generally speaking, FDWs can move under external effects, and this case needs special consideration. The review of the theoretical formalism presented in this section is based on work [17]. The typical geometry of a GFET with a 2D-graphene layer(channel)-on-ferroelectric film with $180^{\circ}-\mathrm{FDW}$ is shown in Fig. 8. The top gate is deposited on an oxide layer and a graphene sheet is separated from a ferroelectric film by an ultra-thin paraelectric dead layer, which emerges due to several reasons such as an incomplete polarization screening at the surface and an imperfect process of graphene deposition on ferroelectric. There are various types of such layers treated

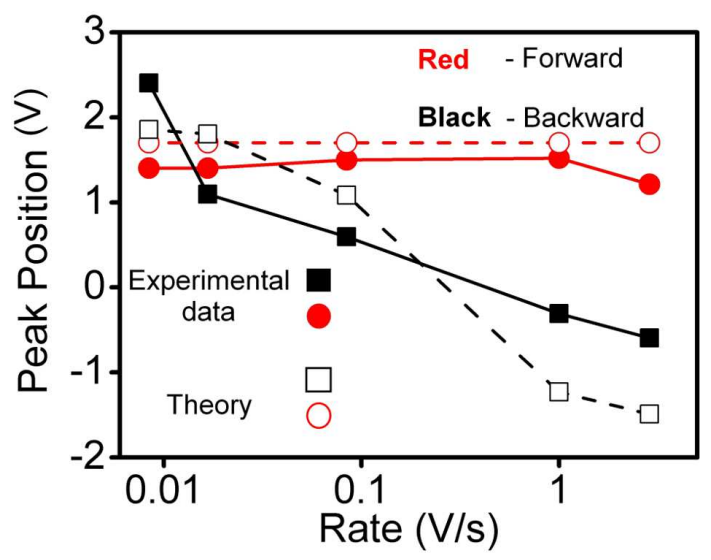

Fig. 7. Experimental [48] and theoretical dependences of the electro-neutrality point position on the gate voltage sweeping rate for the GFET on the PZT ferroelectric substrate Reproduced from work [A.N. Morozovska, A.I. Kurchak, M.V. Strikha. Phys. Rev. Appl. 8, 054004 (2017)] with permission from AIP Publishing

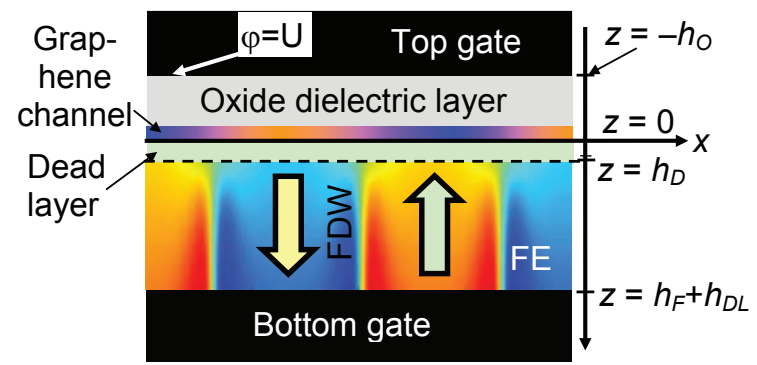

Fig. 8. Schematic diagram of $180^{\circ}$-ferroelectric domain walls (FDWs) in the "top gate-oxide dielectric layer-graphene channel-paraelectric dead layer-ferroelectric film-bottom gate" heterostructure. The ferroelectric substrate is in a perfect electric contact with the bottom gate electrode. Adapted from [A.I. Kurchak, E.A. Eliseev, S.V. Kalinin, M.V. Strikha, A.N. Morozovska. Phys. Rev. Appl. 8, 024027 (2017)] with permission from AIP Publishing for the authors' artwork

as ultrathin ones and located under the surface of the ferroelectric substrate, where the spontaneous polarization is absent (or negligibly small) because of the surface contamination, zero extrapolation length, and/or strong depolarization field $[49,50]$. A periodic voltage applied to the top gate can induce the FDWs to move in the ferroelectric substrate.

The single-layer graphene is regarded to be an infinitesimally thin sheet with $2 \mathrm{D}$ densities for the electron, $g_{n}$, and hole, $g_{p}$, states (see, e.g., works [3,38])

$g_{n}(\epsilon)=g_{p}(\epsilon)=2 \epsilon /\left(\pi \hbar^{2} v_{\mathrm{F}}^{2}\right)$. 
Hence, the 2D concentrations of electrons in the conduction band and holes in the valence band of graphene equal

$n_{2 \mathrm{D}}(\varphi)=\int_{0}^{\infty} d \epsilon g_{n}(\epsilon) f\left(\epsilon-E_{\mathrm{F}}-e \varphi\right)$,

and

$p_{2 \mathrm{D}}(\varphi)=\int_{0}^{\infty} d \epsilon g_{p}(\epsilon) f\left(\epsilon+E_{\mathrm{F}}+e \varphi\right)$,

respectively, where, $E_{\mathrm{F}}$ is the Fermi energy level. The graphene charge density is equal to

$\sigma_{G}(\psi)=e\left(p_{2 \mathrm{D}}(\psi)-n_{2 \mathrm{D}}(\psi)\right)$,

where

$\psi=\frac{e \varphi+E_{\mathrm{F}}}{k_{\mathrm{B}} T}$.

The equations of state $\mathbf{D}=\epsilon_{0} \epsilon_{\mathrm{O}} \mathbf{E}$ and $\mathbf{D}=\epsilon_{0} \epsilon_{\mathrm{DL}} \mathbf{E}$, where $\epsilon_{0}$ is the universal dielectric constant, relate the electrical displacement $\mathbf{D}$ and the electric field $\mathbf{E}$ in the oxide dielectric and ultrathin dead layers (their thicknesses are $h_{\mathrm{O}}$ and $h_{\mathrm{DL}}$, respectively) to each other. The relative dielectric permittivity of the dead layer $\epsilon_{\mathrm{DL}} \sim 10^{2}$ [51] is rather high. The potential $\varphi_{\text {DL }}$ inside the dead layer satisfies the Laplace equation. Note that the problem of the dielectric permittivity of a 2D-graphene layer is still under debate (see, e.g., work [52]).

The ferroelectric film has the thickness $l$, and the ferroelectric polarization $P_{3}^{\mathrm{f}}$ in the $180^{\circ}$ domain wallsurface junctions is directed along the polar axis $z$ (Fig. 8). The polarization $z$-component equals

$P_{3}\left(r, E_{3}\right)=P_{3}^{\mathrm{f}}\left(r, E_{3}\right)+\epsilon_{0}\left(\epsilon_{33}^{\mathrm{b}}-1\right) E_{3}$,

where the so-called relative "background" permittivity $\epsilon_{i j}^{\mathrm{b}}$ [49] is introduced. The values of $\epsilon_{i j}^{\mathrm{b}}$ are not related to a soft ferroelectric mode, being limited by the linear dielectric response of the lattice. Therefore, for the majority of ferroelectrics perovskites, those values fall within an interval of 4-7 (as for their experimental determination, see work [53] and references therein). The spatial distribution of the ferroelectric polarization $P_{3}(x, y, z)$ is determined from the timedependent LGD-type Euler-Lagrange equation

$\Gamma \frac{\partial P_{3}}{\partial t}+a P_{3}+b P_{3}^{3}+c P_{3}^{5}-g \Delta P_{3}=E_{3}$, where $\Gamma$ is the Landau-Khalatnikov relaxation coefficient [41], $g$ is a gradient coefficient, and $\Delta$ stands for the 3D Laplace operator. The constants $a=$ $=\alpha_{T}\left(T-T_{C}\right), b$, and $c$ are coefficients of the LGD potential series expansion in the polarization; they are also called the linear, $a$, and nonlinear, $b$ and $c$, dielectric stiffness coefficients. The corresponding boundary conditions are of the third kind [54] and include the extrapolation lengths $\Lambda_{ \pm}$[55].

For the problem geometry shown in Fig. 8, the corresponding system of electrostatic equations can be found in work [17]. The relevant boundary conditions are as follows: fixed potentials at the top $\left(z=-h_{\mathrm{O}}\right)$ and bottom $\left(z=h_{\mathrm{DL}}+h_{\mathrm{F}} \approx h_{\mathrm{F}}\right)$ gate electrodes; the continuity of the electric potential across the graphene layer $(z=0)$; the relation $D_{3}^{\mathrm{O}}-D_{3}^{\mathrm{DL}}=\sigma_{G}(x, y)$, where $\sigma_{G}(x, y)$ is the surface charges in graphene, for the normal electric displacement components $D_{3}^{\mathrm{O}}=\epsilon_{0} \epsilon_{\mathrm{O}} E_{3}$ and $D_{3}^{\mathrm{DL}}=\epsilon_{0} \epsilon_{\mathrm{DL}} E_{3}$ at $z=0$; and the continuity of the normal displacement component across the dead layer/ferroelectric interface, $D_{3}^{\mathrm{f}}=D_{3}^{\mathrm{DL}}$, where $D_{3}^{\mathrm{f}}=\epsilon_{0} \epsilon_{33}^{\mathrm{b}} E_{3}+P_{3}^{\mathrm{f}}$ and $D_{3}^{\mathrm{DL}}=\epsilon_{0} \epsilon_{\mathrm{DL}} E_{3}$. The gate voltage is periodic with a period $T_{g}: U(t)=U_{\max } \sin \left(2 \pi t / T_{g}\right)$.

For the domains to move, the ferroelectric film thickness should exceed a critical value $l_{c r}$ for the sizeinduced phase transition into the paraelectric phase, with the parameter $l_{c r}$ depending on the thicknesses of the dielectric and dead layers [56-58]. Domains appear above the critical thickness, since they minimize the depolarization field energy in the gap and dielectric layer [36].

Since a typical lateral dimension of the ferroelectric film, $L_{\mathrm{FE}}$, is much larger than the graphene channel length $L$, an odd or even number of domain walls can pass along the channel during the gate voltage oscillation period $T_{g}$, depending on the interrelation between the graphene channel length $L$ and the pe$\operatorname{riod} T_{\mathrm{FE}}$ of the domain structure in the ferroelectric film (see work [17]). The realistic situations can be modeled by periodic, mixed or antiperiodic boundary conditions (BCs) for the polarization component $P_{3}$ and its derivative $\frac{\partial P_{3}}{\partial x}$, as well as the electric potential $\varphi_{f}$ and its derivative $\frac{\partial \varphi_{f}}{\partial x}$, at the lateral boundaries $x= \pm L / 2$ [17].

Using the results of works [15,17], for the even $(2 k)$ number of domain walls in the graphene channel between the source and drain electrodes, the channel conductances $G_{+}^{\text {total }}$ and $G_{-}^{\text {total }}$ are identical for both 
gate voltage polarities: $G_{+}^{\text {total }}=G_{-}^{\text {total }}$. It is so because, at each polarity, there are $k p-n$ junctions with their conductance described by Eq. (8) from work [15], and $k p-n$ junctions with their conductance described by Eq. (10) from work [15]. For the odd $(2 k+1)$ number of walls, Eq. (14) from work [15] can be modified:

$\frac{G_{+}^{\text {total }}}{G_{-}^{\text {total }}}=\frac{\beta(L+\lambda)+\lambda k(1+\beta)}{\beta(L+\lambda)+\lambda k(1+\beta)+\lambda}$,

where the factor $\beta$ beta is defined by Eq. (2.5). A typical electron mean free path $\lambda$ is essentially smaller that the channel length $L$.

In a pronounced diffusion regime for the current $(\beta L \gg \lambda)$ and provided a large number of the walls, i.e. if $k \gg k_{c r}$, where

$k_{c r}=1+\frac{\beta L}{(1+\beta) \lambda}$,

the graphene channel conductance is given by the expression [3, 38]

$G=\frac{\lambda\left(n_{2 \mathrm{D}}\right)}{L} \frac{2 e^{2}}{\hbar \pi^{3 / 2}} W \sqrt{n_{2 \mathrm{D}}}$.

The mean free path $\lambda\left(n_{2 \mathrm{D}}\right) \sim \sqrt{n_{2 \mathrm{D}}}$ for the scattering at ionized centers in the substrate and in the temperature interval far from the Curie temperature [33].

\subsection{Analysis of correlated dynamics for ferroelectric domains and $p-n$ junctions}

In this section, we present the results of numerical simulation of the problem concerned. The parameters used in calculations are listed in Table I of work [17]. The component $P_{3}$ of the ferroelectric film polarization, variation of the $2 \mathrm{D}$ concentration of free charge carriers in the graphene channel $\Delta n_{G}=\left(p_{2 \mathrm{D}}-n_{2 \mathrm{D}}\right)$, and effective charge carrier ratio

$\Delta \eta\left(U_{\max }\right)=\frac{\Delta n_{G}\left(+U_{\max }\right)}{\Delta n_{G}\left(-U_{\max }\right)}$

were calculated as functions on the periodic gate voltage $U(t)=U_{\max } \sin \left(2 \pi t / T_{g}\right)$. The hysteresis loops for the average polarization $P_{3}(U)$ and the concentration variation $\Delta n_{G}(U)$ are shown in Fig. 9 .

At relatively low voltages $\left(U_{\max } \leq 2 \mathrm{~V}\right)$, the polarization and concentration loops have a quasielliptic shape [black curves in Figs. 9, $a-d$. This shape is a result of the polydomain structure of the film and due to the motion of the domain walls under the action

ISSN 2071-0194. Ukr. J. Phys. 2018. Vol. 63, No. 1 of the electric field, when the gate voltage changes between $-U_{\max }$ and $+U_{\max }$. As the gate voltage amplitude $U_{\max }$ increases to $5 \div 10 \mathrm{~V}$, the domain walls start to collide, and the domains with the opposite polarization orientations almost mutually "annihilate" at definite periodic time moments $t$; afterward, the polarized state of the film with a certain degree of unipolarity partially restores (see red and magenta curves in Figs. 9, $a-d$ ). The loop asymmetry and the rectification ratio are associated with the symmetry of $\mathrm{BCs}$ for the polarization and electric potential at the lateral surfaces $x= \pm L / 2$.

Completely symmetric loops for the polarization $P_{3}(U)$ and the concentration variation $\Delta n_{G}(U)$ correspond to periodic BCs (Figs. 9, $a$ and $c$ ). The rectification effect is absent in the case of periodic BCs. It is so because the effective ratio $\Delta \eta\left(U_{\max }\right) \equiv-1$ for all $U_{\max }($ Fig. 9,e), since the even number of domain walls are available in the ferroelectric substrate at any moment. The characteristic distributions of the polarization component $P_{3}$ and the free charge concentration along the graphene channel are shown in Figs. 10, $a$ and 10, $c$, respectively. Two $p-n$ junctions are induced by two moving domain walls at certain moments during each gate voltage period.

The antiperiodic BCs lead to asymmetric loops in the polarization, $P_{3}(U)$, and concentration, $\Delta n_{G}(U)$, dependences (Figs. 9, $b$ and 9, $d$, respectively). The vertical asymmetry and the horizontal shift of the $P_{3}(U)$ loop are much stronger than the corresponding parameters for the $\Delta n_{G}(U)$ loop, because the polarization acts indirectly on the charge via the depolarization field. The asymmetry of the $P_{3}(U)$ and $\Delta n_{G}(U)$ dependences becomes weaker, as the maximum voltage increases (cf. different loops in Figs. 9, $c$ and 9, $f$ ). The rectification effect is evident at low and moderate voltages, since the effective ratio $\Delta \eta\left(U_{\max }\right) \ll 1$ at $1<U_{\max }<4$. At higher voltages (Fig. $9, f)$, the ratio saturates, $\Delta \eta\left(U_{\max }\right) \rightarrow-1$, because an odd number of domain walls mostly move in the ferroelectric film. The characteristic distributions of the polarization and graphene charge are shown in Figs. $10, b$ and $10, d$, respectively. Three $p-n$ junctions are induced by three moving domain walls during one gate voltage period.

\subsection{Extrinsic size effect}

Note that the conductance of the graphene channel, which is proportional to the variation of the to- 

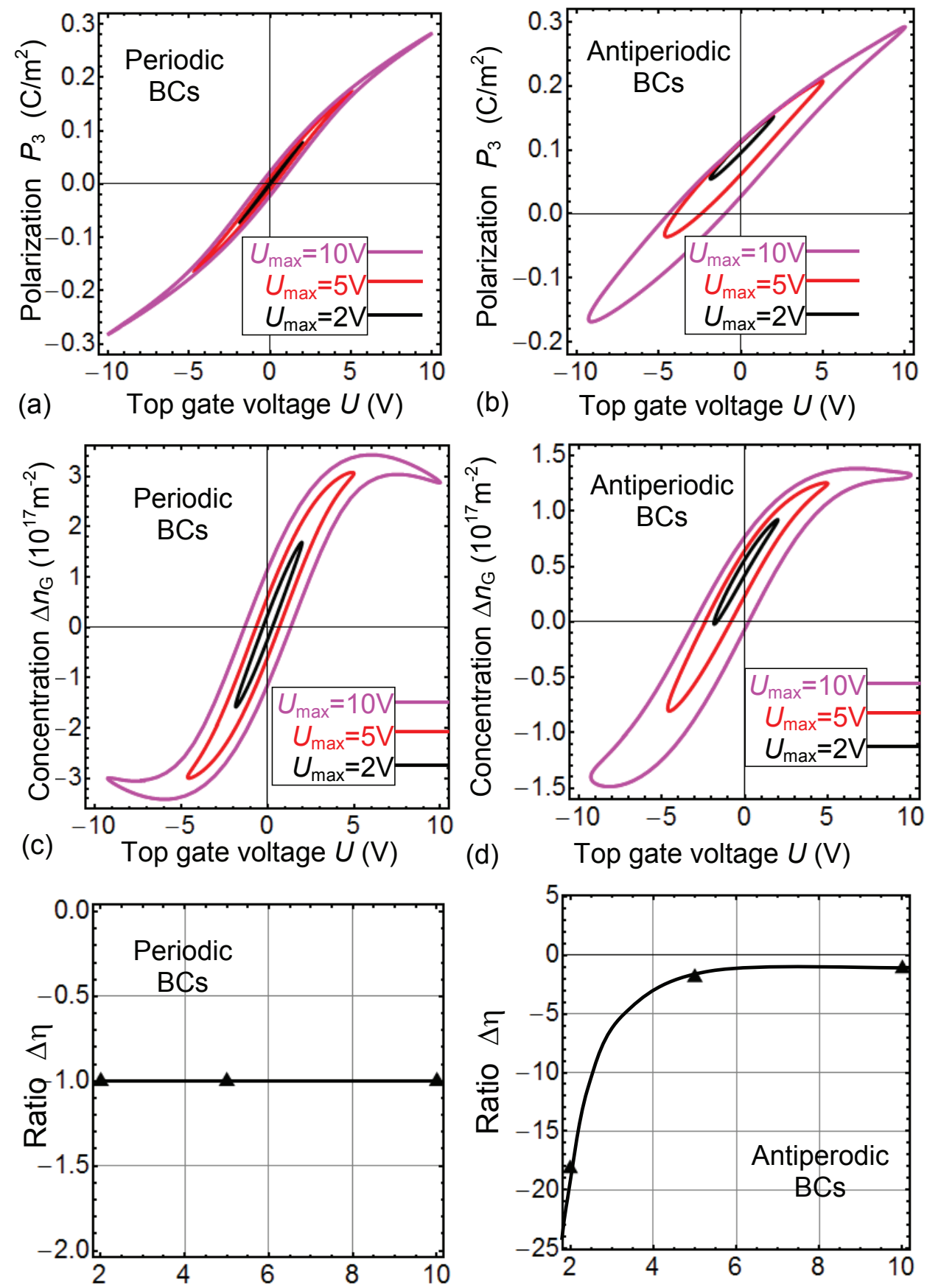

(e) Voltage amplitude $U_{\max }(\mathrm{V})$

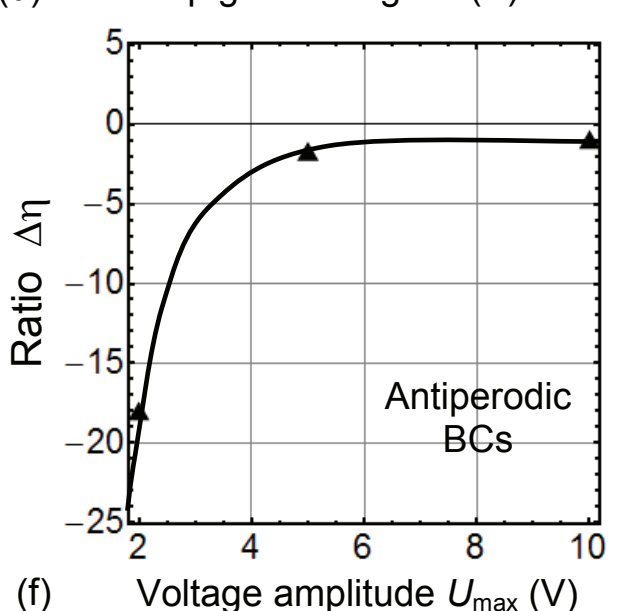

Fig. 9. Hysteresis loops of the ferroelectric polarization $P_{3}(U)(a, b)$, charge carriers concentration variations in the graphene channel $\Delta n_{G}(U)(c, d)$, and conductance ratio $\Delta \eta\left(U_{\max }\right)(e, f)$ calculated for periodic $(a, c, e)$ and antiperiodic $(b, d, f)$ boundary conditions (BCs). $(a, b, c, d)$ Black, red, and magenta loops correspond to different gate voltage amplitudes $U_{\text {max }}=2$, 5 , and $10 \mathrm{~V}$, respectively. The gate voltage period $T_{g}=10^{3}$ s. Adapted from [A.I. Kurchak, E.A. Eliseev, S.V. Kalinin, M.V. Strikha, A.N. Morozovska. Phys. Rev. Appl. 8, 024027 (2017)] with permission from AIP Publishing for the authors' artwork 

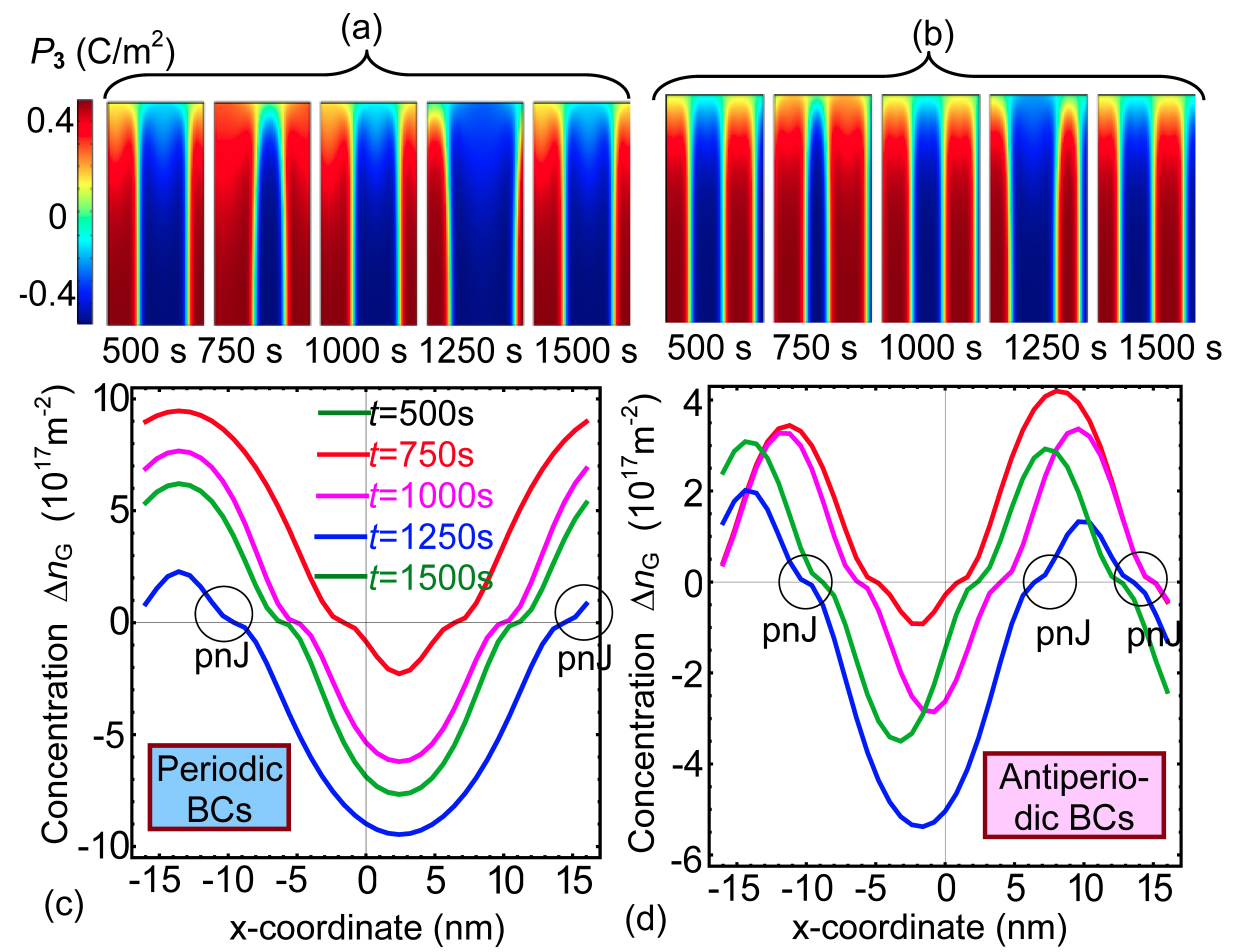

Fig. 10. Spatial distributions of the polarization component $P_{3}(a, b)$ and the 2D-concentration of the free charge carriers $\Delta n_{G}$ $(c, d)$ in the ferroelectric film at time moments $t=500,750,1000,1250$, and $1500 \mathrm{~s}$ (indicated in the plot) within a single gate-voltage period $T_{g}=10^{3} \mathrm{~s}$ for periodic $(a, c)$ and antiperiodic $(b, d)$ BCs. The gate voltage amplitude $U_{\text {max }}=5 \mathrm{~V}$. The other parameters are the same as in Fig. 5. The transient process, which vanishes rather rapidly, is not shown, and the dependences are shown since $t=500 \mathrm{~s}$. Adapted from [A.I. Kurchak, E.A. Eliseev, S.V. Kalinin, M.V. Strikha, A.N. Morozovska. Phys. Rev. Appl. 8, 024027 (2017)] with permission from AIP Publishing for the authors' artwork

tal charge carrier concentration $\Delta n_{G}(L, t)$, depends on its length $L$ (see the color map of the concentration variation $\Delta n_{G}(L, t)$ average over the channel length in the "channel length $L$ versus time $t$ " coordinates in Fig. 11). The phenomenon, which we called the "extrinsic size effect" [15], consists in a quasiperiodic modulation of the conductivity amplitude with the channel length for periodic BCs. The modulation extremes are the most pronounced at $L \approx 27.5 \mathrm{~nm}$ (the first maxima and minima) and $50 \mathrm{~nm}$ (the second maxima and minima) for the parameters listed in work [15]. The modulation becomes less pronounced as $L$ increases (cf. the contrast between the modulation maxima and minima at $L \approx 27.5$ and $50 \mathrm{~nm}$ ). Furthermore, the distance $\Delta L$ between the maxima is voltageindependent and slightly increases together with $L$. We expect that the extrinsic size effect should disappear for channels, which are much longer than

ISSN 2071-0194. Ukr. J. Phys. 2018. Vol. 63, No. 1 the intrinsic period of a domain structure in the ferroelectric substrate.

\section{Graphene Separation and Stretching by Piezoelectric Effect in Ferroelectric Domains}

The $p-n$ junctions in graphene on ferroelectric substrates were actively studied for rather a long time, but the influence of the piezoelectric effect in the ferroelectric substrate with FDWs on graphene characteristics has been considered only recently. Namely, as was shown in work [18], an elastic strain can significantly affect the graphene conductance via stretching the graphene surface and separating graphene sections at the steps between the elongated and shoterned domains. The theoretical formalism presented in this section is based on work [18]. 


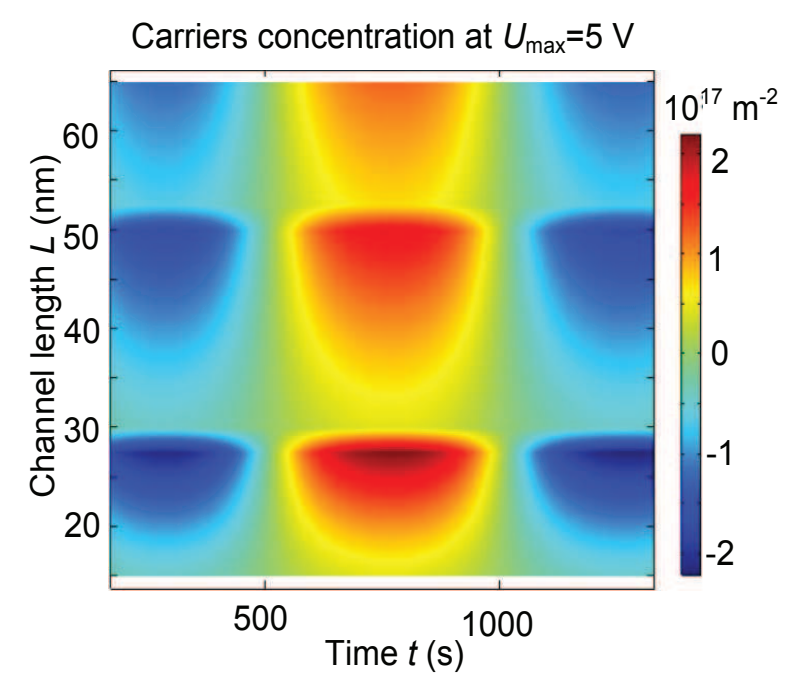

Fig. 11. Dependence of the electron concentration on the channel length and the time for periodic BCs and the gate voltage amplitude $U_{\max }=5 \mathrm{~V}$. Adapted from [A.I. Kurchak, E.A. Eliseev, S.V. Kalinin, M.V. Strikha, A.N. Morozovska. Phys. Rev. Appl. 8, 024027 (2017)] with permission from AIP Publishing for the authors' artwork

The idea consists in that ferroelectric domain stripes with opposite spontaneous polarizations elongate or shorten due to the piezo-effect, depending on the voltage polarity applied to the substrate. If the voltage is applied to the gate of the GFeFET with FDWs, one domain elongates and the next one shortens (Fig. 12). The surface displacement can be significant for ferroelectrics with high piezoelectric coefficients. For instance, for $\mathrm{PbZr}_{0.5} \mathrm{Ti}_{0.5} \mathrm{O}_{3}(\mathrm{PZT})$, those piezoelectric coefficients can reach $0.3-1 \mathrm{~nm} / \mathrm{V}$ depending on the film thickness and the temperature [59]. The corresponding displacement step $h$ at the piezoelectric surface is about $0.5-1 \mathrm{~nm}$ for a gate voltage of about $1-3 \mathrm{~V}$. The thickness $d \leq 0.5 \mathrm{~nm}$ of the physical gap between the graphene and ferroelectric layers is governed by the van der Waals interaction. The binding energy density $J$ for graphene on a $\mathrm{SiO}_{2}$ substrate is about $0.5 \mathrm{~J} / \mathrm{m}^{2}$ [60]. Since the graphene adhesion to the $\mathrm{SiO}_{2}$ surface should be the strongest among other surfaces, it is natural to expect that the $J$-value for graphene on the PZT surface would be smaller. Young's modulus $Y$ of graphene is giant: it equals $1 \mathrm{TPa}[61,62]$. Under such conditions, a partially separated piece of the graphene layer appears at the step when the normal component $F_{n}$ of the elastic tension force
$F$ applied to a carbon atom exceeds the force $F_{b}$ binding this atom to the surface (see the force scheme in Fig. 12, b). As a result, the separated graphene section, which has the length $l+\Delta l$ and is located between the bound sections, becomes "suspended".

\subsection{Piezoelectric displacement of ferroelectric substrate surface}

An analytical expression for the vertical displacement $u_{3}(x)$ of the separated graphene section in a vicinity of the FDW-surface junction was derived in work [18] in the framework of the decoupling approximation [63-67]. The displacement $u_{3}(x)$ looks like [18]

$u_{3}(x)=-U\left[W_{33}(x) d_{33}+W_{31}(x) d_{31}\right]$,

where $U$ is the voltage difference between the top and bottom electrodes, i.e. the gate voltage; and $d_{33}$ and $d_{31}$ are piezoelectric coefficients. Specific expressions for $W_{33}(x)$ and $W_{31}(x)$ are given in work [18].

Figure 13 shows typical profiles of the PZT surface displacement. The values of the PZT film thickness $H$ are varied in the interval $H=20 \div 500 \mathrm{~nm}$, and the separation gap $d=0.5 \mathrm{~nm}$. One can see that a step emerging at the FDW-surface junction is the widest for the smallest ratio $d / H$ (curve 1 ) and becomes essentially thinner, as this ratio increases (curves 2 to 4 ). It is also evident that the maximum height $h$ of the step changes non-monotonically, as the $d / H$ ratio increases, but the difference between the displacements far from the domain wall,

$h_{\infty}=\left|u_{3}(x \rightarrow \infty)-u_{3}(x \rightarrow-\infty)\right|$,

is the same for all curves and is given by the expression [18]

$h_{\infty}=2|U|\left[d_{33}+(1+2 \nu) d_{31}\right]$

A complete separation (i.e. exfoliation) of graphene induced by the piezoelectric effect is hardly possible for thick ferroelectric films with smooth profiles of the surface displacement corresponding to curve 1 in Fig. 13. The exfoliation becomes impossible if there is only one domain wall in the film, and the graphene sheet is only stretched by the piezoelectric effect. The partial separation or complete exfoliation of graphene 


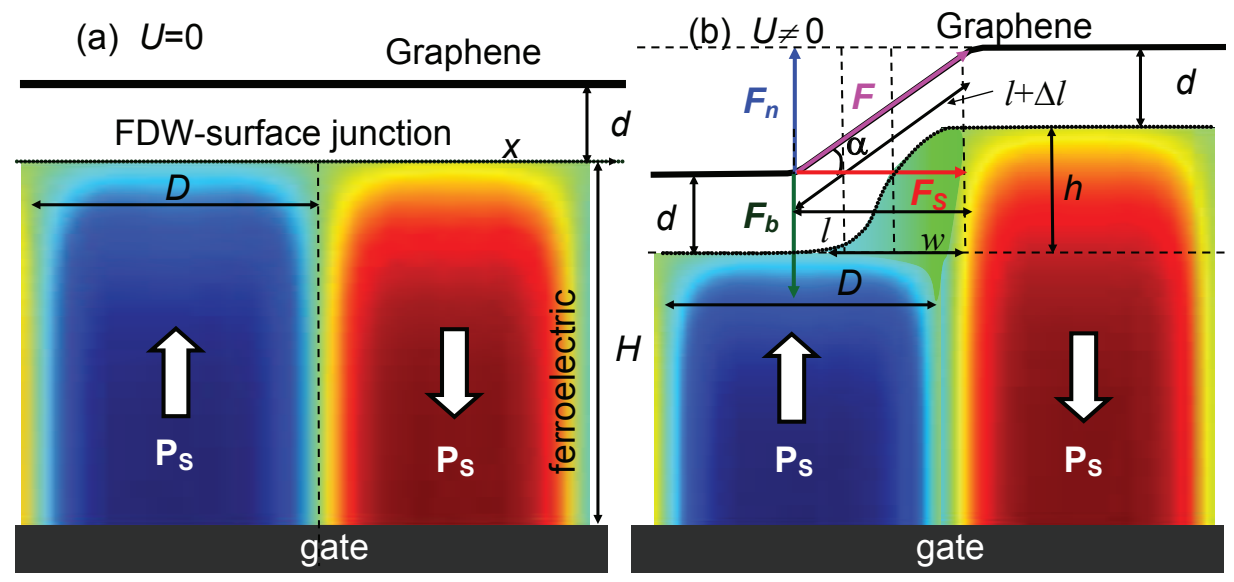

Fig. 12. Partial separation of a graphene channel section induced by the piezoelectric effect at the FDW-surface junction. The separation is absent at the zero applied voltage, $U=0(a)$, and appears at a nonzero one, $U \neq 0(b)$. $F$ is the elastic tension force, $F_{n}$ its normal component, $F_{S}$ its lateral component, and $F_{b}$ the force binding a carbon atom to the surface. Adapted from [A.N. Morozovska, A.I. Kurchak, M.V. Strikha. Phys. Rev. Appl. 8, 054004 (2017)] with permission from AIP Publishing for the authors' artwork

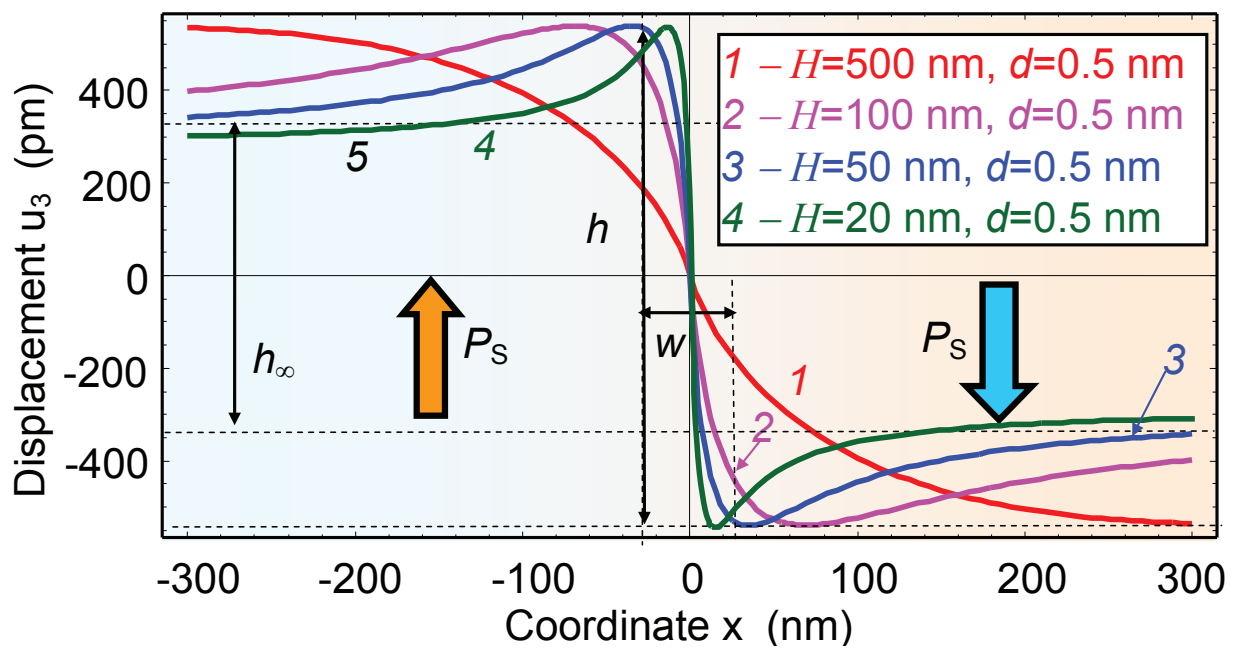

Fig. 13. Profiles of the ferroelectric surface displacement $u_{3}$ calculated for various ferroelectric film thicknesses $H$ and distances $d$ (indicated in the legend). The gate voltage $U=1 \mathrm{~V}$. The thermodynamic piezoelectric coefficients $d_{33} \approx 10^{3} \mathrm{pm} / \mathrm{V}$ and $d_{31} \approx-450 \mathrm{pm} / \mathrm{V}$, and Poisson's ratio $v=0.3$ correspond to PZT at room temperature. Note that the vertical picometer scale is much smaller than the horizontal nanometer scale. Adapted from [A.N. Morozovska, A.I. Kurchak, M.V. Strikha. Phys. Rev. Appl. 8, 054004 (2017)] with permission from AIP Publishing for the authors' artwork

is preferable when the latter contacts with a ferroelectric surface with relatively sharp profiles across the FDWs (Fig. 13, curves 2 to 4 ).

\subsection{Graphene separation, stretching, and exfoliation: impact on the conductance}

Taking into account that the normal component $F_{n}$ of the tangential force $F$ and the binding force $F_{b}$ are ISSN 2071-0194. Ukr. J. Phys. 2018. Vol. 63, No. 1 equal to each other (see Fig. 12, b), we derived [18] the following analytical expression for the minimum length $l$ of a separated graphene section:

$l=h \sqrt[3]{\frac{Y d}{2 J}}>|U|\left(d_{33}+(1+2 \nu) d_{31}\right) \sqrt[3]{\frac{4 Y d}{J}}$.

The indicated inequality originates from the inequality $h>h_{\infty}$ (see Fig. 13]. Estimates made with 
Eq. (5.3) showed that the stretched section length can reach tens of nanometers for the gate voltages $|U| \geq 3 \mathrm{~V}$, provided the PZT parameters at room temperature, the binding energies $J<0.25 \mathrm{~J} / \mathrm{m}^{2}$, and the separation gap $d=0.5 \mathrm{~nm}$.

The graphene channel conductance $G$ in the diffusion regime can change significantly, because electrons in the separated stretched section are scattered by acoustic phonons [38]. The voltage dependence $G(U)$ for the graphene channel of the length $L$, when its section $l(U)$ is separated and the remaining section $L-l(U)$ is bound, obeys the Matthiessen rule [38]

$G(U)=W\left[\frac{L-l(U)}{\sigma_{B}}+\frac{l(U)}{\sigma_{S}}\right]^{-1}$.

The separated section length $l(U)=\chi|U|$, where the coefficient

$\chi=\left(d_{33}+(1+2 \nu) d_{31}\right) \sqrt[3]{4 Y d / J}$

in accordance with work [18].

The conductivity of the remaining bound part looks like [3]

$\sigma_{B}=\frac{2 e^{2}}{\pi^{3 / 2} \hbar} \lambda_{B} \sqrt{n_{2 \mathrm{D}}}$,

where $\hbar$ Planck's constant, $\lambda_{B}$ the electron mean free path in the bound part of graphene channel. The concentration $n_{2 \mathrm{D}}$ of $2 \mathrm{D}$ electrons far from the FDWs can be regarded as a constant voltage-independent value, namely, $n_{2 \mathrm{D}} \approx\left|P_{S} / e\right|[17,18,33]$. Then, for the most common case of electron scattering in the graphene channel at ionized impurities in the substrate, $\lambda_{B}=$ $=\xi \sqrt{n_{2 \mathrm{D}}}$, where the proportionality coefficient $\xi$ depends on the substrate material and graphene-ferroelectric interface chemistry. From Eq. (5.5), we obtain the dependence

$\sigma_{B}\left(n_{2 \mathrm{D}}\right)=\frac{2 e^{2} \xi}{\pi^{3 / 2} \hbar} n_{2 \mathrm{D}} \approx 8.75 \times 10^{-5} \xi n_{2 \mathrm{D}} \mathrm{S}$.

Taking into account that the $P_{S}$-value for thin films can be one order of magnitude smaller than in the bulk, the concentration varies in the interval $n_{2 \mathrm{D}} \cong$ $\cong(0.3 \div 3) \times 10^{18} \mathrm{~m}^{-2}$ depending on the film thickness. But it should be regarded as a voltage- and coordinate-independent constant far from the FDW. Thus, elementary estimates give $\sigma_{B} \cong(0.15 \div 15) \times$ $\times 10^{-3} \Omega^{-1}$ for reasonable values $\lambda_{B}=10 \div 100 \mathrm{~nm}$ and $P_{S}=0.05 \div 0.5 \mathrm{C} / \mathrm{m}^{2}$.
Electron collisions with acoustic phonons are the main channel for the electron scattering in the separated stretched section of structurally perfect graphene. In this case, $\lambda_{S}(E) \sim 1 / E[3]$, and we arrive at the well-known paradox: the conductivity $\sigma_{S}$ should not depend on the $2 \mathrm{D}$ electron concentration in the graphene channel. For estimations, the wellknown upper limit for $\sigma_{S}$ [3] can be used:

$\sigma_{S}=\frac{4 e^{2} \hbar \rho_{m} v_{\mathrm{F}}^{2} v_{S}^{2}}{\pi D_{A}^{2} k_{\mathrm{B}} T}$.

Here, $\rho_{m} \approx 7.6 \times 10^{-7} \mathrm{~kg} / \mathrm{m}^{2}$ is the $2 \mathrm{D}$ mass density of charge carriers in graphene, $v_{S} \approx 2.1 \times 10^{4} \mathrm{~m} / \mathrm{s}$ is the sound velocity in graphene, $k_{\mathrm{B}}=1.38 \times 10^{-23} \mathrm{~J} / \mathrm{K}$ is the Boltzmann constant, and $D_{A} \approx 19 \mathrm{eV}$ is the acoustic deformation potential that describes the electron-phonon interaction. For the indicated parameters and room temperature, Eq. (5.7) yields $\sigma_{S} \approx 3.4 \times 10^{-2} \Omega^{-1}$.

Figure 14 illustrates the conductance $G$ calculated for various values of the gate voltage $U$, binding energy $J$, and channel length $L$. The conductance increases together with $U$; the corresponding growth is monotonic and faster than linear, being the most pronounced at small binding energies $J \leq 0.2 \mathrm{~J} / \mathrm{m}^{2}$ (Fig. 14, a) and small channel lengths $L \leq 100 \mathrm{~nm}$ (Fig. 14, b). The conductance ratio $G(U) / G(0)$ does not exceed 1.25 in the case of partial graphene separation (curves 1 ) for realistic parameters. However, it can be significantly larger, if the domain stripe period $D$ is much shorter than the channel length $L$. Let the length of the separated section be approximately equal to half the channel length, i.e. $l \approx L / 2$. Provided that the $p-n$ junctions at FDWs do not change the general conductance of the graphene channel significantly, the electron mean free paths $\lambda_{B} \ll D$ and $\lambda_{S} \ll D$, so that $\sigma_{S} \gg \sigma_{B}$, and Eq. (5.4) yields $G(U) / G(0) \approx$ $\approx 2$. Note that the ratio $G(U) / G(0)$ can be significantly larger than 2 , e.g., in the case of mostly suspended graphene $(l \approx L)$ with $\sigma_{S} \gg \sigma_{B}$. However, the possibility of this limiting case needs a special examination.

Figure 15 schematically illustrates the conductance $G(U)$ calculated using Eqs. (5.4)-(5.7). The predicted effect of conductance modulation can be very useful in order to improve and miniaturize various electronic devices (such as advanced logic elements, memory cells, highly efficient hybrid electrical modula-

ISSN 2071-0194. Ukr. J. Phys. 2018. Vol. 63, No. 1 

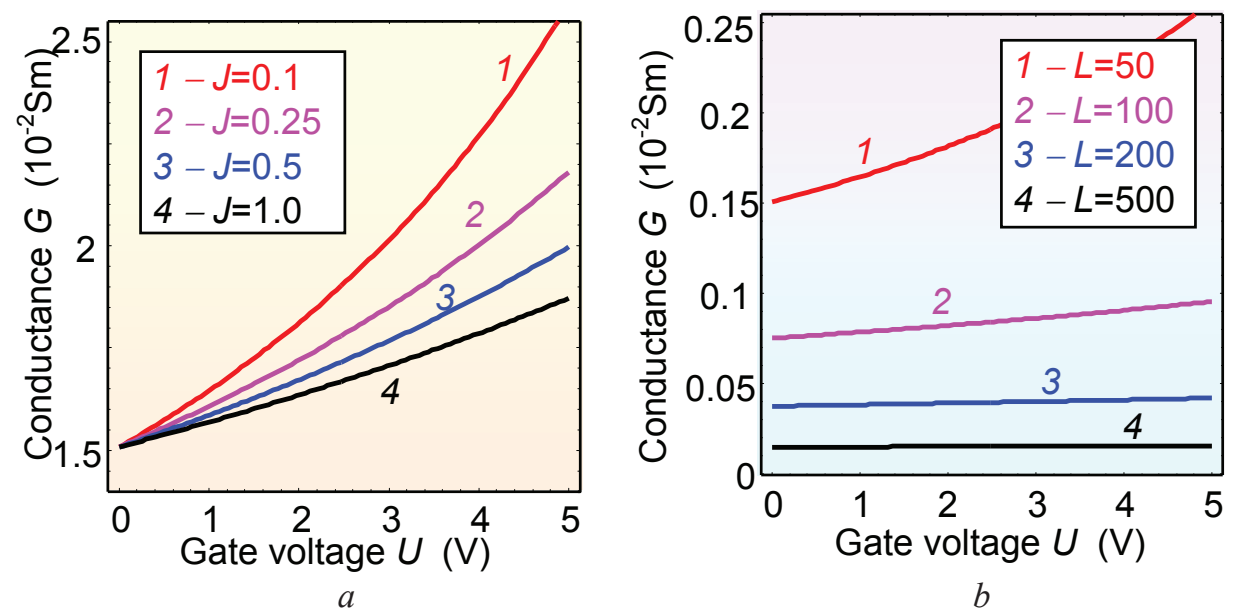

Fig. 14. Dependences of the conductance $G$ on the gate voltage $U$ calculated $(a)$ for various binding energies $J$ (indicated in the legend in $\mathrm{J} / \mathrm{m}^{2}$ units) at $L=50 \mathrm{~nm}$ and $(b)$ for various channel lengths $L$ (indicated in the legend in $\mathrm{nm}$ units) at $J=0.5 \mathrm{~J} / \mathrm{m}^{2}$. Adapted from [A.N. Morozovska, A.I. Kurchak, M.V. Strikha. Phys. Rev. Appl. 8, 054004 (2017)] with permission from AIP Publishing for the authors' artwork

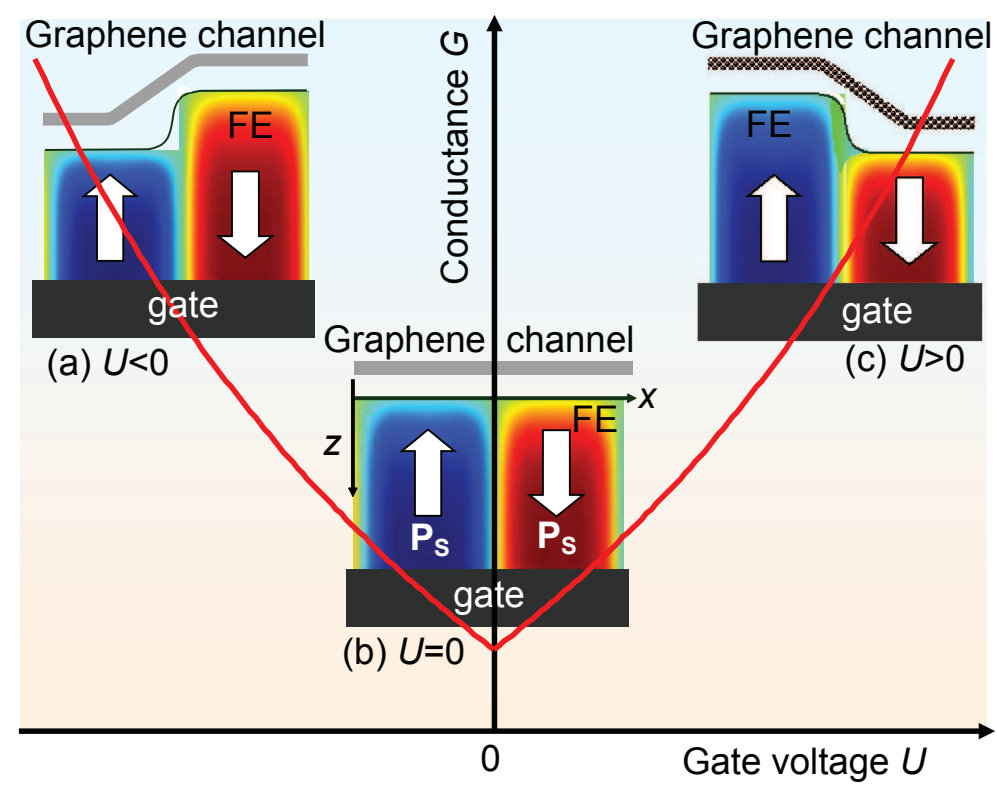

Fig. 15. Modulation of the graphene channel conductance by the piezo-effect in the GFeFET. The plot schematically demonstrates the dependence of the channel conductance $G$ on the gate voltage $U$. Insets $(a)$ and $(c)$ illustrate the vertical piezoelectric displacement of the ferroelectric surface at the FDW, which causes a partial separation of graphene sections induced by negative and positive gate voltages, respectively. The displacement and the corresponding graphene separation are absent at $U=0$ [inset (b)]. Adapted from [A.N. Morozovska, A.I. Kurchak, M.V. Strikha. Phys. Rev. Appl. 8, 054004 (2017)] with permission from AIP Publishing for the authors' artwork

tors and transducers of voltage-to-current type, and piezo-resistive elements). We also propose an alternative method to create suspended graphene sections, which is based on the piezo-effect in the ferro- electric substrate. The method does not require any additional technological procedures like the chemical etching or mechanical treating of the substrate surface. 
It is the graphene channel sections separated by piezo-effect that can invoke physical effects that are interesting for fundamental physics. First, the graphene channel conductance changes significantly in the diffusion regime, because electrons in the stretched section are scattered by acoustic phonons. Second, mechanic vibrations in the $\mathrm{MHz}$ interval can be realized here [68]. Third, high pseudo-magnetic fields were reported for stretched graphene [69].

\section{Conclusions}

Since GFETs on the ferroelectric substrate are promising candidates for novel non-volatile ultra-fast ferroelectric memory (FRAM) units [46, 47], the theoretical description of the operation of those devices requires reliable knowledge of the ferroelectric response in a wide frequency interval, as well as the nature of the charge carrier trapping processes at a given frequency.

It has been shown that the contact between the domain wall and the ferroelectric surface creates a $p$ $n$ junction in the graphene channel. The charge carrier concentration induced at that in graphene by uncompensated ferroelectric dipoles can reach a value of $10^{19} \mathrm{~m}^{-2}$ in the ballistic regime, which is two orders of magnitude higher than the values obtained for graphene on non-ferroelectric substrates [14]. The performance of the majority of realistic graphene devices depends on the electron mean free path (about 50-250 nm), and, hence, they operate in the diffusion regime. We present the theory of the conductance of $p-n$ junctions in the graphene channel located on the ferroelectric substrate with FDWs. The consideration is valid for an arbitrary current regime: from ballistic to diffusion one [15]. In works [14,15], it was demonstrated that graphene channels with $p-n$ junctions at the FDWs can serve as excellent rectifiers because of their large conductance ratio for the "direct" and "inverse" voltages applied between the source and drain electrodes, which is a result of a high ferroelectric substrate permittivity.

The competition between the ferroelectric dipoles and the dipoles adsorbed on the graphene surface determines the FRAM operating characteristics. We proposed a general theory for versatile hysteretic phenomena in the graphene-based field effect transistor (GFET), which allows an analytical description. The theory makes allowance for the existence of external dipoles on the graphene free surface and localized states at the graphene-surface interface [16]. We demonstrated that the adsorbed dipole molecules (e.g., dissociated or highly polarized water molecules) can induced a hysteresis loop in the dependence of the charge carrier concentration on the gate voltage and in the corresponding gatevoltage dependence of the graphene conductance in the GFET on substrates of various types, including the most common $\mathrm{SiO}_{2}$ and ferroelectric ones. The results of work [16], although being valid for the description of hysteretic phenomena in various realistic GFETs that operate at low and intermediate frequencies, may be not applicable directly to describe the ferroelectric response at ultra-high frequencies; first of all, because the high-frequency response of adsorbed and ferroelectric dipoles requires further experimental studies. The results obtained can be useful when predicting the most suitable ferroelectric substrates for graphene-on-ferroelectric structures used in ultrafast non-volatile memory units of new generation.

Using a self-consistent approach based on the Landau-Ginzburg-Devonshire phenomenology and classical electrostatics, we studied the dynamics of $p$ $n$ junctions in the graphene channel induced by the nucleation, motion, and reversal of stripe domains in the ferroelectric substrate [17]. It was demonstrated that, in the case of perfect electric contact between the ferroelectric and the graphene sheet, relatively low gate voltages are required to induce a pronounced hysteresis in the dependences of ferroelectric polarization and graphene charge on the gate voltage. The electrostatic boundary conditions for the polarization at the lateral surfaces of the ferroelectric substrate govern the conductance asymmetry of the graphene channel between the source and drain electrodes. We also revealed a pronounced extrinsic size effect in the dependence of the graphene channel conductance on the channel length.

The $p-n$ junctions at FDWs in graphene have been actively studied recently, but the role of the piezoelectric effect in the ferroelectric substrate has not been considered. We proposed [18] a piezoelectric mechanism to control the conductance of the GFET on the ferroelectric substrate with immobile domain walls. In particular, we predicted that the graphene channel conductance can be controlled by the gate voltage due to the piezoelectric elongation

ISSN 2071-0194. Ukr. J. Phys. 2018. Vol. 63, No. 1 
and shortening of ferroelectric domains with opposite polarization directions. At the same time, the gate voltage can create bound, separated, suspended, and stretched sections of the graphene single layer with significantly different conductance values. Our calculations demonstrated a capability to substantially (by several times) increase the conductance of GFETs on ferroelectric substrates with a high piezoelectric response. We also proposed an alternative method to fabricate suspended graphene sections, which is based on the piezo-effect in the ferroelectric substrate. The method does not require any additional technological procedures like the chemical etching or mechanical treating of the substrate surface.

Taking into account that the conductance of the graphene-on-ferroelectric structure is significantly higher than that of graphene on ordinary dielectric substrates, the predicted effect can be very useful for the improvement and miniaturization of many types of electronic devices, including various logic elements, memory cells, highly efficient hybrid electrical modulators and voltage-to-current transducers with the frequency doubling and relatively low operational voltages, and piezo-resistive elements.

1. K. Novoselov, A. Geim, S. Morozov, D. Jiang, Y. Zhang, S. Dubonos, I. Grigorieva, A. Firsov. Electric field effect in atomically thin carbon films. Science 306, 666 (2004).

2. A. Geim. Graphene: status and prospects. Science 324, 1530 (2009).

3. S. Das Sarma, Shaffique Adam, E.H. Hwang, E. Rossi. Electronic transport in two-dimensional graphene. Rev. Mod. Phys. 83, 407 (2011).

4. B. Amorim, A. Cortijo, F. de Juan, A.G. Grushin, F. Guinea, A. Gutierrez-Rubio, H. Ochoa, V. Parente, R. Roldan, P. San-Jose, J. Schiefele, M. Sturla, M.A.H. Vozmediano. Novel effects of strains in graphene and other two dimensional materials. Phys. Rep. 617, 1 (2016).

5. Gerardo G.Naumis, Salvador Barraza-Lopez, Maurice Oliva-Leyva, Humberto Terrones. A review of the electronic and optical properties of strained graphene and other similar 2D materials. arXiv:1611.08627 (2016).

6. Yi Zheng, Guang-Xin Ni, Chee-Tat Toh, Chin-Yaw Tan, Kui Yao, Barbaros Özyilmaz. Graphene field-effect transistors with ferroelectric gating. Phys. Rev. Lett. 105, 166602 (2010).

7. Woo Young Kim, Hyeon-Don Kim, Teun-Teun Kim, HyunSung Park, Kanghee Lee, Hyun Joo Choi, Seung Hoon Lee, Jaehyeon Son, Namkyoo Park, Bumki Min. Grapheneferroelectric metadevices for nonvolatile memory and reconfigurable logic-gate operations. Nature Commun. 7, 10429 (2016).
8. X. Hong, J. Hoffman, A. Posadas, K. Zou, C.H. Ahn, J. Zhu. Unusual resistance hysteresis in $n$-layer graphene field effect transistors fabricated on ferroelectric $\mathrm{Pb}\left(\mathrm{Zr}_{0.2} \mathrm{Ti}_{0.8}\right) \mathrm{O}_{3}$. Appl. Phys. Lett. 97, 033114 (2010).

9. A. Rajapitamahuni, J. Hoffman, C.H. Ahn, X. Hong. Examining graphene field effect sensors for ferroelectric thin film studies. Nano Lett. 13, 4374 (2013).

10. M. Humed Yusuf, B. Nielsen, M. Dawber, X. Du. Extrinsic and intrinsic charge trapping at the graphene/ferroelectric interface. Nano Lett. 14, 5437 (2014).

11. J.H. Hinnefeld, Ruijuan Xu, S. Rogers, Shishir Pandya, Moonsub Shim, L.W. Martin, N. Mason. Single gate $p n$ junctions in graphene-ferroelectric devices. arXiv: 1506.07138 (2015).

12. C. Baeumer, D. Saldana-Greco, J.M.P. Martirez, A.M. Rappe, M. Shim, L.W. Martin. Ferroelectrically driven spatial carrier density modulation in graphene. Nature Commun. 6, 6136 (2015).

13. Jie, Wenjing, Jianhua Hao. Time-dependent transport characteristics of graphene tuned by ferroelectric polarization and interface charge trapping. Nanoscale 10, 328 (2017).

14. A.N. Morozovska, E.A. Eliseev, M.V. Strikha. Ballistic conductivity of graphene channel with $p-n$ junction on ferroelectric domain wall. Appl. Phys. Lett. 108, 232902 (2016).

15. M.V. Strikha, A.N. Morozovska. Limits for the graphene on ferroelectric domain wall $p-n$-junction rectifier for different regimes of current. J. Appl. Phys. 120, 214101 (2016).

16. A.I. Kurchak, A.N. Morozovska, M.V. Strikha. Hysteretic phenomena in GFET: general theory and experiment. J. Appl. Phys. 122, 044504 (2017).

17. A.I. Kurchak, E.A. Eliseev, S.V. Kalinin, M.V. Strikha, A.N. Morozovska. $p-n$ junctions dynamics in graphene channel induced by ferroelectric domains motion. Phys. Rev. Appl. 8, 024027 (2017).

18. A.N. Morozovska, A.I. Kurchak, M.V. Strikha. Graphene exfoliation at ferroelectric domain wall induced by piezoeffect: impact on the conduction of graphene channel. Phys. Rev. Appl. 8, 054004 (2017).

19. J.R. Williams, L. Di Carlo, C.M. Marcus. Quantum Hall effect in a gate-controlled $p-n$ junction of graphene. Science 317, 638 (2007).

20. V. Cheianov, V. Falko. Selective transmission of Dirac electrons and ballistic magnetoresistance of $n-p$ junctions in graphene. Phys. Rev. B 74, 041403 (2006).

21. J.R. Whyte, J.M. Gregg. A diode for ferroelectric domainwall motion. Nature Commun. 6, 7361 (2015).

22. N.M. Zhang, M.M. Fogler. Nonlinear screening and ballistic transport in a graphene $p-n$ junction. Phys. Rev. Lett. 100, 116804 (2008).

23. Yu.A. Kruglyak, M.V. Strikha. Generalized LandauerDatta-Lundstrom model in application to transport phenomena in graphene. Ukr. J. Phys. Reviews 10, 3 (2015). 
24. C.W. Beenakker. Andreev reflection and Klein tunneling in graphene. Rev. Mod. Phys. 80, 1337 (2008).

25. M.I. Katsnelson, K.S. Novoselov, A.K. Geim. Chiral tunnelling and the Klein paradox in graphene. Nature Phys. 2, 620 (2006).

26. V.V. Cheianov, V.I. Falko, B.L. Altshuler. The focusing of electron flow and a Veselago lens in graphene $p-n$ junctions. Science 315, 1252 (2007).

27. A.N. Morozovska, M.V. Strikha. Pyroelectric origin of the carrier density modulation at graphene-ferroelectric interface. J. Appl. Phys. 114, 014101 (2013).

28. A.N. Morozovska, E.A. Eliseev, A.V. Ievlev, O.V. Varenyk, A.S. Pusenkova, Ying-Hao Chu, V.Ya. Shur, M.V. Strikha, S.V. Kalinin. Ferroelectric domain triggers the charge modulation in semiconductors. J. Appl. Phys. 116, 066817 (2014).

29. I.I. Naumov, A.M. Bratkovsky. Gap opening in graphene by simple periodic inhomogeneous strain. Phys. Rev. B 84, 245444 (2011).

30. T.L. Linnik. Effective Hamiltonian of strained graphene. J. Phys.: Condens. Matter 24, 205302 (2012).

31. T.L. Linnik. Photoinduced valley currents in strained graphene. Phys. Rev. B 90, 075406 (2014).

32. E.A. Eliseev, A.N. Morozovska, S.V. Kalinin, Y.L. Li, Jie Shen, M.D. Glinchuk, L.Q. Chen, V. Gopalan. Surface effect on domain wall width in ferroelectrics. J. Appl. Phys. 106, 084102 (2009).

33. A.N. Morozovska, A.S. Pusenkova, O.V. Varenyk, S.V. Kalinin, E.A. Eliseev, M.V. Strikha. Finite size effects of hysteretic dynamics in multi-layer graphene on ferroelectric. Phys. Rev. B 91, 235312 (2015).

34. S. Datta. Lessons from Nanoelectronics: A New Perspective on Transport (World Scientific, 2015).

35. D. Singh, J.Y. Murthy, T.S. Fisher. Mechanism of thermal conductivity reduction in few-layer graphene. J. Appl. Phys. 110, 094312 (2011).

36. A.K. Tagantsev, L.E. Cross, J. Fousek. Domains in Ferroic Crystals and Thin Films (Springer, 2010).

37. M.V. Strikha. Mechanism of the antihysteresis behavior of the resistivity of graphene on a $\mathrm{Pb}\left(\mathrm{Zr}_{x} \mathrm{Ti}_{1-x}\right) \mathrm{O}_{3}$ ferroelectric substrate. JETP Lett. 95, 198 (2012).

38. A.I. Kurchak, M.V. Strikha. Antihysteresis of the electrical resistivity of graphene on a ferroelectric $\mathrm{Pb}\left(\mathrm{Zr}_{x} \mathrm{Ti}_{1-x}\right) \mathrm{O}_{3}$ substrate. J. Exp. Theor. Phys. 143, 129 (2013).

39. A.I. Kurchak, A.N. Morozovska, M.V. Strikha. Rival mechanisms of hysteresis in the resistivity of graphene channel. Ukr. J. Phys. 58, 472 (2013).

40. Y. Zheng, G.-X. Ni, C.-T. Toh, C.-Y. Tan, K. Yao, B. Özyilmaz. Graphene field-effect transistors with ferroelectric gating. Phys. Rev. Lett. 105, 166602 (2010).

41. L.D. Landau, I.M. Khalatnikov. On the anomalous absorption of sound near a second order phase transition point. Dokl. Akad. Nauk SSSR 96, 469 (1954).

42. S.V. Kalinin, A.N. Morozovska, L.Q. Chen, B.J. Rodriguez. Local polarization dynamics in ferroelectric materials. Rep. Progr. Phys. 73, 056502 (2010).
43. H. Wang, Y. Wu, C. Cong, J. Shang, T. Yu. Hysteresis of electronic transport in graphene transistors. ACS Nano 4, 7221 (2010)

44. M. Lafkioti, B. Krauss, T. Lohmann, U. Zschieschang, H. Klauk, K. Klitzing, J.H. Smet. Graphene on a hydrophobic substrate: doping reduction and hysteresis suppression under ambient conditions. Nano Lett. 10, 1149 (2010).

45. A. Veligura, P.J. Zomer, I.J. Vera-Marun, C. Józsa, P.I. Gordiichuk, B.J. van Wees. Relating hysteresis and electrochemistry in graphene field effect transistors. J. Appl. Phys. 110, 113708 (2011).

46. M.V. Strikha. Non-volatile memory of new generation and ultrafast IR modulators based on graphene on ferroelectric substrate. In Functional Nanomaterials and Devices for Electronics, Sensors and Energy Harvesting, edired by A. Nazarov, F. Balestra, V. Kilchytska, D. Flandre (Springer, 2014), p. 163.

47. M.V. Strikha. Hysteresis in the resistivity of graphene channel. In Chemical Functionalization of Carbon $\mathrm{Na}$ nomaterials: Chemistry and Application, edited by V.K. Thakur, M.K. Thakur (Taylor and Francis, 2015), p. 939.

48. C. Baeumer, S.P. Rogers, R. Xu, L.W. Martin, M. Shim. Tunable carrier type and density in graphene/ $\mathrm{PbZr}_{0.2} \mathrm{Ti}_{0.8} \mathrm{O}_{3}$ hybrid structures through ferroelectric switching. Nano Lett. 13, 1693 (2013).

49. A.K. Tagantsev, G. Gerra. Interface-induced phenomena in polarization response of ferroelectric thin films. J. Appl. Phys. 100, 051607 (2006).

50. A.K. Tagantsev, M. Landivar, E. Colla, N. Setter. Identification of passive layer in ferroelectric thin films from their switching parameters. J. Appl. Phys. 78, 2623 (1995).

51. G. Rupprecht, R.O. Bell. Dielectric constant in paraelectric perovskite. Phys. Rev. 135, A748 (1964).

52. Elton J.G. Santos. Electric field effects on graphene materials. In Exotic Properties of Carbon Nanomatter (Springer, 2015), p. 383.

53. J. Hlinka, P. Máton. Phenomenological model of a $90^{\circ}$ domain wall in $\mathrm{BaTiO}_{3}$-type ferroelectrics. Phys. Rev. B 74, 104104 (2006).

54. R. Kretschmer, K. Binder. Surface effects on phase transitions in ferroelectrics and dipolar magnets. Phys. Rev. B 20, 1065 (1979).

55. Chun-Lin Jia, Valanoor Nagarajan, Jia-Qing He, Lothar Houben, Tong Zhao, Ramamoorthy Ramesh, Knut Urban, Rainer Waser. Unit-cell scale mapping of ferroelectricity and tetragonality in epitaxial ultrathin ferroelectric films. Nature Mater. 6, 64 (2007).

56. A.N. Morozovska, E.A. Eliseev, Nicholas V. Morozovsky, S.V. Kalinin. Ferroionic states in ferroelectric thin films. Phys. Rev. B 95, 195413 (2017).

57. E.A. Eliseev, A.N. Morozovska. General approach to the description of the size effect in ferroelectric nanosystems. J. Mater. Sci. 44, 5149 (2009). 
58. A.N. Morozovska, E.A. Eliseev, S.V. Svechnikov, A.D. Krutov, V.Y. Shur, A.Y. Borisevich, P. Maksymovych, S.V. Kalinin. Finite size and intrinsic field effect on the polar-active properties of ferroelectric semiconductor heterostructures. Phys. Rev. B 81, 205308 (2010).

59. M.J. Haun, E. Furman, S.J. Jang, L.E. Cross. Thermodynamic theory of the lead zirconate-titanate solid solution system. Part V: Theoretical calculations. Ferroelectrics 99, 63 (1989) (see figure 16 and table I).

60. S.P. Koenig, N.G. Boddeti, M.L. Dunn, J.S. Bunch. Ultrastrong adhesion of graphene membranes. Nature Nanotechnol. 6, 543 (2011).

61. A. Politano, G. Chiarello. Probing the Young's modulus and Poisson's ratio in graphene/metal interfaces and graphite: A comparative study. Nano Res. 8, 1847 (2015).

62. Tao Chen, Rebecca Cheung. Mechanical properties of graphene. In Graphene Science Handbook. Mechanical and Chemical Properties (CRC Press, 2016), p. 3.

63. F. Felten, G.A. Schneider, J. Muñoz Saldaña, S.V. Kalinin. Modeling and measurement of surface displacements in $\mathrm{BaTiO}_{3}$ bulk material in piezoresponse force microscopy. J. Appl. Phys. 96, 563 (2004).

64. S.V. Kalinin, E.A. Eliseev, A.N. Morozovska. Materials contrast in piezoresponse force microscopy. Appl. Phys. Lett. 88, 23 (2006).

65. A.N. Morozovska, E.A. Eliseev, S.L. Bravina, S.V. Kalinin. Resolution function theory in piezoresponse force microscopy: Domain wall profile, spatial resolution, and tip calibration. Phys. Rev. B 75, 174109 (2007).

66. S.V. Kalinin, A.N. Morozovska, L.Q. Chen, B.J. Rodriguez. Local polarization dynamics in ferroelectric materials. Rep. Progr. Phys. 73, 056502 (2010).

67. S.V. Kalinin, B.J. Rodriguez, S.-H. Kim, S.-K. Hong, A. Gruverman, E.A. Eliseev. Imaging mechanism of piezoresponse force microscopy in capacitor structures. Appl. Phys. Lett. 92, 152906 (2008).
68. J.S. Bunch, A.M. van der Zande, S.S. Verbridge, I.W. Frank, D.M. Tanenbaum, J.M. Parpia, H.G. Craighead, P.L. McEuen. Electromechanical resonators from graphene sheets. Science 315, 490 (2007).

69. N. Levy, S.A. Burke, K.L. Meaker, M. Panlasigui, A. Zettl, F. Guinea, A.H. Castro Neto, M.F. Crommie. Straininduced pseudo-magnetic fields greater than 300 tesla in graphene nanobubbles. Science 329, 544 (2010).

Received 26.12.17.

Translated from Ukrainian by O.I. Voitenko

М.B. Cmpixa, А.І. Курчак, Г.М. Морозовсъка

ВПЛИВ ДОМЕННОЇ СТРУКТУРИ

СЕГНЕТОЕЛЕКТРИЧНОЇ ПІДКЛАДКИ

НА ПРОВІДНІСТЬ ГРАФЕНУ

(АВТОРСЬКИЙ ОГЛЯД)

$\mathrm{P}$ е з ю м е

Огляд присвячено останнім теоретичним дослідженням впливу доменної структури сегнетоелектричної підкладки на провідність графенового каналу. Розглянутий аналітичний опис ефектів пам'яті гістерезисного типу у польовому транзисторі на основі графена-на-сегнетоелектрику, з урахуванням адсорбованих дипольних шарів на вільній поверхні графену і локалізованих станів на його інтерфейсах. Аналізуються аспекти нещодавно розвинутої теорії провідності $p-n$-переходів у графеновому каналі на сегнетоелектричній підкладці, які створені 180-градусною сегнетоелектричною доменною структурою, причому розглянуті випадки різних режимів струму, від балістичного до дифузійного. Обговорюється вплив розмірних ефектів у таких системах та можливість використання результатів для вдосконалення характеристик польових транзисторів з графеновим каналом, комірок енергонезалежної сегнетоелектричної пам'яті з довільним доступом, сенсорів, а також для мініатюризації різних пристроїв функціональної наноелектроніки. 